Supplement of Biogeosciences, 17, 1169-1180, 2020 https://doi.org/10.5194/bg-17-1169-2020-supplement (C) Author(s) 2020. This work is distributed under the Creative Commons Attribution 4.0 License.

(c) (1)

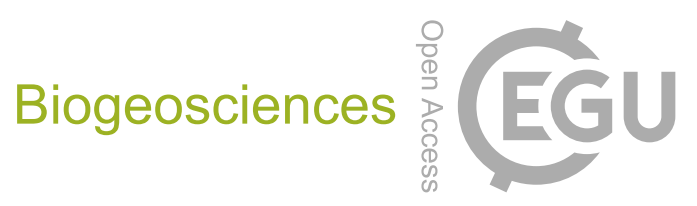

Supplement of

\title{
Light availability modulates the effects of warming in a marine $\mathbf{N}_{2}$ fixer
}

\section{Xiangqi Yi et al.}

Correspondence to: Kunshan Gao (ksgao@xmu.edu.cn)

The copyright of individual parts of the supplement might differ from the CC BY 4.0 License. 
Table S1. The parameters derived from the short-term thermal responses for $\mathrm{N}_{2}$ fixation measured at two light intensity (HL: $160 \mu \mathrm{mol}$ quanta $\mathrm{m}^{-2} \mathrm{~s}^{-1}$, LL: $50 \mu \mathrm{mol}$ quanta $\mathrm{m}^{-2}$ $\mathrm{s}^{-1}$ ) in the Trichodesmium cells acclimated to different levels of temperature and light intensity (Fig. S2); values represent the means \pm the standard deviations of biological replicates $(n=3)$.

\begin{tabular}{|c|c|c|c|c|c|}
\hline & \multirow{3}{*}{ Assay light } & \multicolumn{4}{|c|}{ Acclimation } \\
\hline & & \multicolumn{2}{|c|}{$\mathrm{HL}$} & \multicolumn{2}{|c|}{$\mathrm{LL}$} \\
\hline & & $23^{\circ} \mathrm{C}$ & $27^{\circ} \mathrm{C}$ & $23^{\circ} \mathrm{C}$ & $27^{\circ} \mathrm{C}$ \\
\hline \multirow{2}{*}{$\mathrm{N}\left(\mathrm{T}_{\mathrm{c}}\right)^{*}$} & HL & $39.2 \pm 1.6$ & $39.7 \pm 1.8$ & $26.3 \pm 2.2$ & $17.6 \pm 1.1$ \\
\hline & LL & $30.8 \pm 4.0$ & $32.1 \pm 1.1$ & $19.3 \pm 0.4$ & $15.6 \pm 0.7$ \\
\hline \multirow{2}{*}{$\mathrm{E}_{\mathrm{a}}(\mathrm{eV})$} & HL & $0.54 \pm 0.08$ & $0.94 \pm 0.11$ & $0.88 \pm 0.20$ & $1.15 \pm 0.20$ \\
\hline & LL & $0.31 \pm 0.17$ & $0.48 \pm 0.08$ & $0.43 \pm 0.04$ & $1.01 \pm 0.11$ \\
\hline \multirow{2}{*}{$\mathrm{E}_{\mathrm{h}}(\mathrm{eV})$} & HL & $3.93 \pm 0.70$ & $3.78 \pm 0.58$ & $4.08 \pm 1.24$ & $4.85 \pm 1.11$ \\
\hline & LL & $2.77 \pm 1.03$ & $6.56 \pm 1.52$ & $8.49 \pm 0.96$ & $4.96 \pm 0.84$ \\
\hline \multirow{3}{*}{$\mathrm{T}_{\mathrm{h}}\left({ }^{\circ} \mathrm{C}\right)^{\#}$} & HL & $32.5 \pm 0.3$ & $32.3 \pm 0.4$ & $32.0 \pm 0.6$ & $31.9 \pm 0.5$ \\
\hline & LL & $31.8 \pm 0.8$ & $31.7 \pm 0.2$ & $31.2 \pm 0.1$ & $30.8 \pm 0.2$ \\
\hline & LL & $10.7 \pm 1.1$ & $13.6 \pm 0.5$ & $8.1 \pm 0.5$ & $7.3 \pm 0.3$ \\
\hline
\end{tabular}


Table S2. P values of Tukey's tests for pair-wise comparisons between treatments; HL and LL separately represent light-saturating and light-limiting growth conditions; 23, 27 and 31 separately represent growth temperatures of 23,27 and $31{ }^{\circ} \mathrm{C}$

\begin{tabular}{|c|c|c|c|c|c|c|}
\hline & $\begin{array}{r}\text { Specific growth } \\
\text { rate } \\
\end{array}$ & $\mathrm{N}_{\text {growth }}$ & $\begin{array}{r}\text { Effective } \\
\text { quantum } \\
\text { yield } \\
\end{array}$ & alpha & $E_{k}$ & ETR $_{\text {max }}$ \\
\hline \multicolumn{7}{|l|}{ 27:HL- } \\
\hline 23:HL & $2.62 \mathrm{E}-05$ & 3.70E-06 & 9.69E-01 & 8.27E-01 & $1.00 \mathrm{E}+00$ & $2.65 \mathrm{E}-01$ \\
\hline \multicolumn{7}{|l|}{ 31:HL- } \\
\hline 23:HL & 4.39E-01 & 7.36E-03 & 2.99E-03 & 5.01E-01 & $2.46 \mathrm{E}-01$ & $1.06 \mathrm{E}-05$ \\
\hline \multicolumn{7}{|l|}{ 23:LL- } \\
\hline 23:HL & $1.56 \mathrm{E}-06$ & 1.91E-07 & 2.90E-06 & $2.42 \mathrm{E}-01$ & 4.17E-01 & 9.97E-01 \\
\hline \multicolumn{7}{|l|}{ 27:LL- } \\
\hline $23: \mathrm{HL}$ & $1.54 \mathrm{E}-06$ & 2.87E-07 & $1.52 \mathrm{E}-05$ & 5.33E-01 & $1.00 \mathrm{E}+00$ & $2.24 \mathrm{E}-02$ \\
\hline \multicolumn{7}{|l|}{ 31:LL- } \\
\hline 23:HL & $4.26 \mathrm{E}-07$ & $3.81 \mathrm{E}-09$ & $2.24 \mathrm{E}-07$ & $2.92 \mathrm{E}-03$ & $9.91 \mathrm{E}-01$ & $4.48 \mathrm{E}-07$ \\
\hline \multicolumn{7}{|l|}{ 31:HL- } \\
\hline 27:HL & 3.32E-04 & $1.21 \mathrm{E}-03$ & $1.05 E-02$ & $9.02 \mathrm{E}-02$ & $3.59 \mathrm{E}-01$ & $9.51 \mathrm{E}-07$ \\
\hline \multicolumn{7}{|l|}{ 23:LL- } \\
\hline 27:HL & $2.82 \mathrm{E}-09$ & $2.48 \mathrm{E}-10$ & 6.60E-06 & $3.52 \mathrm{E}-02$ & 2.91E-01 & $1.38 \mathrm{E}-01$ \\
\hline \multicolumn{7}{|l|}{ 27:LL- } \\
\hline 27:HL & 2.80E-09 & 3.34E-10 & 3.85E-05 & $9.92 \mathrm{E}-02$ & $1.00 \mathrm{E}+00$ & $5.55 \mathrm{E}-04$ \\
\hline \multicolumn{7}{|l|}{ 31:LL- } \\
\hline 27:HL & $1.27 \mathrm{E}-09$ & $9.58 \mathrm{E}-12$ & 4.43E-07 & $4.58 \mathrm{E}-04$ & $1.00 \mathrm{E}+00$ & $6.33 \mathrm{E}-08$ \\
\hline \multicolumn{7}{|l|}{ 23:LL- } \\
\hline 31:HL & $2.61 \mathrm{E}-07$ & 7.31E-09 & 1.99E-03 & 9.92E-01 & $1.01 \mathrm{E}-02$ & 1.77E-05 \\
\hline \multicolumn{7}{|l|}{ 27:LL- } \\
\hline $31: H L$ & $2.58 \mathrm{E}-07$ & $9.70 \mathrm{E}-09$ & $2.34 \mathrm{E}-02$ & $1.00 \mathrm{E}+00$ & $2.98 \mathrm{E}-01$ & $1.91 \mathrm{E}-03$ \\
\hline \multicolumn{7}{|l|}{ 31:LL- } \\
\hline 31:HL & $8.25 \mathrm{E}-08$ & $2.51 \mathrm{E}-10$ & 4.31E-05 & $5.85 \mathrm{E}-02$ & 5.10E-01 & 7.59E-02 \\
\hline \multicolumn{7}{|l|}{ 27:LL- } \\
\hline 23:LL & $1.00 \mathrm{E}+00$ & $5.44 \mathrm{E}-01$ & $6.74 \mathrm{E}-01$ & 9.87E-01 & $3.51 \mathrm{E}-01$ & 4.67E-02 \\
\hline \multicolumn{7}{|l|}{ 31:LL- } \\
\hline 23:LL & 7.44E-01 & $1.24 \mathrm{E}-03$ & 1.47E-01 & $1.46 \mathrm{E}-01$ & $1.90 \mathrm{E}-01$ & $6.70 \mathrm{E}-07$ \\
\hline \multicolumn{7}{|l|}{ 31:LL- } \\
\hline 27:LL & 7.53E-01 & $6.09 \mathrm{E}-04$ & $1.23 \mathrm{E}-02$ & $5.31 \mathrm{E}-02$ & $9.98 \mathrm{E}-01$ & $2.51 \mathrm{E}-05$ \\
\hline
\end{tabular}


Table S3. AICc for all tested 'nlme' models

\begin{tabular}{|c|c|c|}
\hline Model Number & Fixed effects structure & $\mathrm{AlCC}$ \\
\hline 569 & $\mathrm{~N}(\mathrm{Tc}) \sim$ Light $*$ Temperature, Ea $\sim$ Temperature, Eh $\sim$ Light + Temperature, Th $\sim$ Light + Temperature & 239.0259 \\
\hline 444 & $\mathrm{~N}(\mathrm{Tc}) \sim$ Light + Temperature, Ea $\sim$ Temperature, Eh $\sim$ Light + Temperature, Th $\sim$ Light + Temperature & 240.8197 \\
\hline 469 & $\mathrm{~N}(\mathrm{Tc}) \sim$ Light + Temperature, Ea $\sim$ Light + Temperature, Eh $\sim$ Light + Temperature, Th $\sim$ Light + Temperature & 242.1449 \\
\hline 194 & $\mathrm{~N}(\mathrm{Tc}) \sim$ Light, Ea $\sim$ Temperature, Eh $\sim$ Light + Temperature, Th $\sim$ Light + Temperature & 242.7849 \\
\hline 219 & $\mathrm{~N}(\mathrm{Tc}) \sim$ Light, Ea $\sim$ Light + Temperature, Eh $\sim$ Light + Temperature, Th Light + Temperature & 242.8497 \\
\hline 217 & $\mathrm{~N}(\mathrm{Tc}) \sim$ Light, Ea $\sim$ Light + Temperature, Eh $\sim$ Light + Temperature, Th $\sim$ Light & 243.6906 \\
\hline 199 & $\mathrm{~N}(\mathrm{Tc}) \sim$ Light, Ea $\sim$ Temperature, Eh $\sim$ Light * Temperature, Th Light + Temperature & 243.7218 \\
\hline 592 & $\mathrm{~N}(\mathrm{Tc}) \sim$ Light * Temperature, Ea $\sim$ Light + Temperature, Eh Light + Temperature, Th Light & 243.8034 \\
\hline 567 & $\mathrm{~N}(\mathrm{Tc}) \sim$ Light * Temperature, Ea $\sim$ Temperature, Eh $\sim$ Light + Temperature, Th Light & 243.8716 \\
\hline 467 & $\mathrm{~N}(\mathrm{Tc}) \sim$ Light + Temperature, Ea $\sim$ Light + Temperature, Eh $\sim$ Light + Temperature, Th $\sim$ Light & 243.9612 \\
\hline 570 & $\mathrm{~N}(\mathrm{Tc}) \sim$ Light * Temperature, Ea $\sim$ Temperature, Eh $\sim$ Light + Temperature, Th Light * Temperature & 244.3424 \\
\hline 192 & $\mathrm{~N}(\mathrm{Tc}) \sim$ Light, Ea $\sim$ Temperature, Eh $\sim$ Light + Temperature, Th $\sim$ Light & 244.5404 \\
\hline 242 & $\mathrm{~N}(\mathrm{Tc}) \sim$ Light, Ea $\sim$ Light $*$ Temperature, Eh $\sim$ Light + Temperature, Th $\sim$ Light & 244.732 \\
\hline 574 & $\mathrm{~N}(\mathrm{Tc}) \sim$ Light $*$ Temperature, Ea $\sim$ Temperature, Eh $\sim$ Light * Temperature, Th $\sim$ Light + Temperature & 245.1117 \\
\hline 224 & $\mathrm{~N}(\mathrm{Tc}) \sim$ Light, Ea $\sim$ Light + Temperature, Eh $\sim$ Light * Temperature, Th $\sim$ Light + Temperature & 245.4236 \\
\hline 442 & $\mathrm{~N}(\mathrm{Tc}) \sim$ Light + Temperature, Ea $\sim$ Temperature, Eh $\sim$ Light + Temperature, Th $\sim$ Light & 245.4466 \\
\hline 560 & $\mathrm{~N}(\mathrm{Tc}) \sim$ Light $*$ Temperature, Ea Temperature, Eh $\sim$ Light, Th $\sim$ Light * Temperature & 245.4972 \\
\hline 222 & $\mathrm{~N}(\mathrm{Tc}) \sim$ Light, Ea $\sim$ Light + Temperature, Eh $\sim$ Light * Temperature, Th $\sim$ Light & 246.8219 \\
\hline 244 & $\mathrm{~N}(\mathrm{Tc}) \sim$ Light, Ea $\sim$ Light * Temperature, Eh $\sim$ Light + Temperature, Th $\sim$ Light + Temperature & 247.0617 \\
\hline 619 & $\mathrm{~N}(\mathrm{Tc}) \sim$ Light * Temperature, Ea Light * Temperature, Eh $\sim$ Light + Temperature, Th Light + Temperature & 247.2577 \\
\hline 599 & $\mathrm{~N}(\mathrm{Tc}) \sim$ Light * Temperature, Ea $\sim$ Light + Temperature, Eh $\sim$ Light * Temperature, Th Light + Temperature & 247.4894 \\
\hline 449 & $\mathrm{~N}(\mathrm{Tc}) \sim$ Light + Temperature, Ea $\sim$ Temperature, Eh $\sim$ Light * Temperature, Th $\sim$ Light + Temperature & 247.513 \\
\hline 472 & $\mathrm{~N}(\mathrm{Tc}) \sim$ Light + Temperature, Ea $\sim$ Light + Temperature, Eh $\sim$ Light * Temperature, Th $\sim$ Light & 247.8428 \\
\hline 220 & $\mathrm{~N}(\mathrm{Tc}) \sim$ Light, Ea $\sim$ Light + Temperature, Eh $\sim$ Light + Temperature, Th Light * Temperature & 248.3354 \\
\hline 492 & $\mathrm{~N}(\mathrm{Tc}) \sim$ Light + Temperature, Ea $\sim$ Light * Temperature, Eh $\sim$ Light + Temperature, Th $\sim$ Light & 248.6068 \\
\hline
\end{tabular}


$\mathrm{N}(\mathrm{Tc}) \sim$ Light, Ea $\sim$ Light * Temperature, Eh $\sim$ Light * Temperature, Th $\sim$ Light 249.3418

$\mathrm{N}(\mathrm{Tc}) \sim$ Light + Temperature, Ea $\sim$ Temperature, Eh $\sim$ Light + Temperature, Th $\sim$ Light * Temperature 250.5733

$\mathrm{N}(\mathrm{Tc}) \sim$ Light, Ea $\sim$ Temperature, Eh Light + Temperature, Th Light * Temperature 250.7164

$\mathrm{N}(\mathrm{Tc}) \sim$ Light, Ea $\sim$ Temperature, Eh $\sim$ Light * Temperature, Th $\sim$ Light * Temperature 250.8311

$\mathrm{N}(\mathrm{Tc}) \sim$ Light $*$ Temperature, Ea $\sim$ Temperature, Eh $\sim$ Light * Temperature, Th $\sim$ Light * Temperature 250.9195

$\mathrm{N}(\mathrm{Tc}) \sim$ Light + Temperature, Ea $\sim$ Temperature, Eh $\sim$ Light * Temperature, Th $\sim$ Light * Temperature 251.5586 $\mathrm{N}(\mathrm{Tc}) \sim$ Light + Temperature, Ea $\sim$ Light * Temperature, Eh $\sim$ Light * Temperature, Th $\sim$ Light + Temperature 252.2151

$\mathrm{N}(\mathrm{Tc}) \sim$ Light + Temperature, Ea $\sim$ Temperature, Eh $\sim$ Light * Temperature, Th $\sim$ Light 252.4349

$\mathrm{N}(\mathrm{Tc}) \sim$ Light, Ea $\sim$ Light * Temperature, Eh $\sim$ Light * Temperature, Th $\sim$ Light * Temperature 252.6475 $\mathrm{N}(\mathrm{Tc}) \sim$ Light * Temperature, Ea $\sim$ Light * Temperature, Eh $\sim$ Light * Temperature, Th $\sim$ Light + Temperature 253.2656

$\mathrm{N}(\mathrm{Tc}) \sim$ Light $*$ Temperature, Ea $\sim$ Temperature, Eh $\sim$ Light * Temperature, Th $\sim$ Light 253.3408 $\mathrm{N}(\mathrm{Tc}) \sim$ Light + Temperature, Ea $\sim$ Light + Temperature, Eh $\sim$ Light * Temperature, Th $\sim$ Light * Temperature 253.532

$\mathrm{N}(\mathrm{Tc}) \sim$ Light, Ea $\sim$ Temperature, Eh $\sim$ Light * Temperature, Th Light 255.4939

$\mathrm{N}(\mathrm{Tc}) \sim$ Light * Temperature, Ea $\sim$ Light + Temperature, Eh $\sim 1, \mathrm{Th} \sim$ Light * Temperature 255.8671

$\mathrm{N}(\mathrm{Tc}) \sim$ Light * Temperature, Ea $\sim$ Light * Temperature, Eh $\sim$ Light, Th $\sim$ Light * Temperature 255.9221 $\mathrm{N}(\mathrm{Tc}) \sim$ Light + Temperature, Ea $\sim$ Light * Temperature, Eh $\sim$ Light + Temperature, Th $\sim$ Light * Temperature 257.3548

$\mathrm{N}(\mathrm{Tc}) \sim$ Light + Temperature, Ea $\sim$ Light + Temperature, Eh $\sim$ Light + Temperature, Th 1258.0351

$\mathrm{N}(\mathrm{Tc}) \sim$ Light * Temperature, Ea $\sim$ Temperature, Eh $\sim 1$, Th Light * Temperature 259.8718

$\mathrm{N}(\mathrm{Tc}) \sim$ Light + Temperature, Ea $\sim$ Light + Temperature, Eh $\sim$ Light + Temperature, Th $\sim$ Temperature 260.0544 $\mathrm{N}(\mathrm{Tc}) \sim$ Light + Temperature, Ea $\sim$ Light * Temperature, Eh $\sim$ Light * Temperature, Th $\sim$ Light * Temperature 260.2084

$\mathrm{N}(\mathrm{Tc}) \sim$ Light * Temperature, Ea Light + Temperature, Eh Light + Temperature, Th 1260.4277

$\mathrm{N}(\mathrm{Tc}) \sim$ Light $*$ Temperature, Ea $\sim$ Light * Temperature, Eh $\sim 1$, Th $\sim$ Light $*$ Temperature 262.0768

$\mathrm{N}(\mathrm{Tc}) \sim$ Light + Temperature, Ea Light + Temperature, Eh Light * Temperature, Th 1262.0942

$\mathrm{N}(\mathrm{Tc}) \sim$ Light * Temperature, Ea Light + Temperature, Eh Light + Temperature, Th Temperature 263.3582

$\mathrm{N}(\mathrm{Tc}) \sim$ Light, Ea $\sim$ Light + Temperature, Eh Light + Temperature, Th $\sim$ Temperature 263.4558

$\mathrm{N}(\mathrm{Tc}) \sim$ Light + Temperature, Ea $\sim$ Light + Temperature, Eh Light * Temperature, Th $\sim$ Temperature 263.8886

$\mathrm{N}(\mathrm{Tc}) \sim$ Light, Ea $\sim$ Light + Temperature, Eh Light + Temperature, Th $\sim 1263.9131$

$\mathrm{N}(\mathrm{Tc}) \sim$ Light * Temperature, Ea $\sim$ Light * Temperature, Eh $\sim$ Light, Th $\sim$ Light 264.074

$\mathrm{N}(\mathrm{Tc}) \sim$ Light + Temperature, Ea $\sim$ Light * Temperature, Eh $\sim$ Light + Temperature, Th 1264.1562 
$\mathrm{N}(\mathrm{Tc}) \sim$ Light * Temperature, Ea $\sim$ Light + Temperature, Eh $\sim$ Light, Th $\sim$ Light * Temperature 264.7503 $\mathrm{N}(\mathrm{Tc}) \sim$ Light + Temperature, Ea Light * Temperature, Eh $\sim$ Light + Temperature, Th Temperature $\mathrm{N}(\mathrm{Tc}) \sim$ Light * Temperature, Ea $\sim$ Light + Temperature, Eh $\sim$ Light * Temperature, Th $\sim 1$ $\mathrm{N}(\mathrm{Tc}) \sim$ Light, Ea $\sim$ Light + Temperature, Eh $\sim$ Light * Temperature, Th $\sim$ Temperature $\mathrm{N}(\mathrm{Tc}) \sim$ Light * Temperature, Ea $\sim$ Light + Temperature, Eh Light, Th Light $\mathrm{N}(\mathrm{Tc}) \sim$ Light, Ea $\sim$ Light + Temperature, Eh $\sim$ Light * Temperature, Th $\sim 1$

$\mathrm{N}(\mathrm{Tc}) \sim$ Light $*$ Temperature, Ea $\sim$ Temperature, Eh $\sim$ Light, Th $\sim$ Light

$\mathrm{N}(\mathrm{Tc}) \sim$ Light * Temperature, Ea $\sim$ Temperature, Eh $\sim$ Light, Th $\sim$ Light + Temperature $\mathrm{N}(\mathrm{Tc}) \sim$ Light + Temperature, Ea $\sim$ Light * Temperature, Eh $\sim$ Light, Th $\sim$ Light

$\mathrm{N}(\mathrm{Tc}) \sim$ Light * Temperature, Ea $\sim$ Light + Temperature, Eh $\sim$ Light, Th $\sim$ Light + Temperature $\mathrm{N}(\mathrm{Tc}) \sim$ Light + Temperature, Ea Light + Temperature, Eh Light, Th Light

$\mathrm{N}(\mathrm{Tc}) \sim$ Light * Temperature, Ea $\sim$ Light + Temperature, Eh $\sim 1$, Th $\sim$ Light

$\mathrm{N}(\mathrm{Tc}) \sim$ Light * Temperature, Ea $\sim 1$, Eh $\sim$ Light + Temperature, Th $\sim$ Light * Temperature $\mathrm{N}(\mathrm{Tc}) \sim$ Light, Ea $\sim$ Light * Temperature, Eh $\sim$ Light, Th Light

$\mathrm{N}(\mathrm{Tc}) \sim$ Light + Temperature, Ea $\sim$ Light + Temperature, Eh $\sim$ Light, Th $\sim$ Light + Temperature $\mathrm{N}(\mathrm{Tc}) \sim$ Light + Temperature, Ea $\sim$ Temperature, Eh $\sim$ Light, Th $\sim$ Light * Temperature $\mathrm{N}(\mathrm{Tc}) \sim$ Light, Ea $\sim$ Light + Temperature, Eh $\sim$ Light, Th $\sim$ Light + Temperature

$\mathrm{N}(\mathrm{Tc}) \sim$ Light + Temperature, Ea $\sim$ Temperature, Eh $\sim$ Light, Th $\sim$ Light + Temperature $\mathrm{N}(\mathrm{Tc}) \sim$ Light, Ea $\sim$ Temperature, Eh $\sim$ Light, Th Light + Temperature $\mathrm{N}(\mathrm{Tc}) \sim$ Light + Temperature, Ea $\sim$ Light * Temperature, Eh $\sim$ Light * Temperature, Th $\sim 1$ $\mathrm{N}(\mathrm{Tc}) \sim$ Light $*$ Temperature, Ea $\sim$ Light + Temperature, Eh $\sim$ Light $*$ Temperature, Th $\sim$ Temperature

$\mathrm{N}(\mathrm{Tc}) \sim$ Light * Temperature, Ea $\sim$ Light + Temperature, Eh $\sim 1$, Th $\sim$ Light + Temperature $\mathrm{N}(\mathrm{Tc}) \sim$ Light, Ea $\sim$ Light + Temperature, Eh Light, Th Light

$\mathrm{N}(\mathrm{Tc}) \sim$ Light + Temperature, Ea $\sim$ Temperature, Eh $\sim$ Light, Th $\sim$ Light $\mathrm{N}(\mathrm{Tc}) \sim$ Light, Ea $\sim$ Temperature, Eh $\sim$ Light, Th Light

$\mathrm{N}(\mathrm{Tc}) \sim$ Light, Ea $\sim$ Light * Temperature, Eh $\sim$ Light + Temperature, Th $\sim$ Temperature $\mathrm{N}(\mathrm{Tc}) \sim$ Light * Temperature, Ea $\sim$ Temperature, Eh $\sim$ Temperature, Th $\sim$ Light * Temperature $\mathrm{N}(\mathrm{Tc}) \sim$ Light, Ea $\sim$ Light * Temperature, Eh $\sim$ Light + Temperature, Th $\sim 1$ 
$\mathrm{N}(\mathrm{Tc}) \sim$ Light * Temperature, Ea Light * Temperature, Eh Light + Temperature, Th Temperature 270.6032 $\mathrm{N}(\mathrm{Tc}) \sim$ Light, Ea Light * Temperature, Eh Light, Th Light + Temperature 270.6621

$\mathrm{N}(\mathrm{Tc}) \sim$ Light + Temperature, Ea Light * Temperature, Eh Light * Temperature, Th $\sim$ Temperature 270.771 $\mathrm{N}(\mathrm{Tc}) \sim$ Light * Temperature, Ea $\sim$ Temperature, Eh Temperature, Th Light + Temperature 271.4946

$\mathrm{N}(\mathrm{Tc}) \sim$ Light * Temperature, Ea $\sim$ Temperature, Eh 1, Th Light + Temperature 271.9846 $\mathrm{N}(\mathrm{Tc}) \sim$ Light + Temperature, Ea Light + Temperature, Eh Light, Th Light * Temperature 272.0024 $\mathrm{N}(\mathrm{Tc}) \sim$ Light, Ea $\sim$ Temperature, Eh $\sim$ Light, Th $\sim$ Light * Temperature $\quad 272.172$

$\mathrm{N}(\mathrm{Tc}) \sim$ Light + Temperature, Ea $\sim$ Light * Temperature, Eh $\sim$ Light, Th $\sim$ Light + Temperature 272.4508 $\mathrm{N}(\mathrm{Tc}) \sim$ Light, Ea $\sim$ Light * Temperature, Eh $\sim$ Light * Temperature, Th Temperature 272.5289

$\mathrm{N}(\mathrm{Tc}) \sim$ Light * Temperature, Ea Light * Temperature, Eh Light * Temperature, Th $\sim 1272.9735$

$\mathrm{N}(\mathrm{Tc}) \sim$ Light, Ea $\sim$ Light + Temperature, Eh Light, Th Light * Temperature 272.9841

$\mathrm{N}(\mathrm{Tc}) \sim$ Light, Ea $\sim$ Light * Temperature, Eh Light* Temperature, Th 1272.9892

$\mathrm{N}(\mathrm{Tc}) \sim$ Light * Temperature, Ea $\sim$ Light + Temperature, Eh $\sim$ Temperature, Th $\sim$ Light 273.1855

$\mathrm{N}(\mathrm{Tc}) \sim$ Light * Temperature, Ea Light * Temperature, Eh Light + Temperature, Th $\sim 1 \quad 273.2357$

$\mathrm{N}(\mathrm{Tc}) \sim$ Light * Temperature, Ea Light * Temperature, Eh Light + Temperature, Th Light 273.2932

$\mathrm{N}(\mathrm{Tc}) \sim$ Light * Temperature, Ea 1, Eh $\sim$ Light * Temperature, Th Light* Temperature $\quad 274.258$

$\mathrm{N}(\mathrm{Tc}) \sim$ Light + Temperature, Ea $\sim$ Temperature, Eh $\sim$ Temperature, Th $\sim$ Light + Temperature 274.9348

$\mathrm{N}(\mathrm{Tc}) \sim$ Light, Ea $\sim$ Temperature, Eh $\sim$ Temperature, Th Light + Temperature 275.167

$\mathrm{N}(\mathrm{Tc}) \sim$ Light * Temperature, Ea $\sim$ Light + Temperature, Eh $\sim$ Temperature, Th $\sim$ Light + Temperature 275.8792

$\mathrm{N}(\mathrm{Tc}) \sim$ Light + Temperature, Ea $\sim 1$, Eh $\sim$ Light * Temperature, Th Light + Temperature 275.9204 $\mathrm{N}(\mathrm{Tc}) \sim$ Light, Ea 1, Eh Light + Temperature, Th Light 275.9975

$\mathrm{N}(\mathrm{Tc}) \sim$ Light * Temperature, Ea $\sim 1$, Eh $\sim$ Light + Temperature, Th Light 276.1471

$\mathrm{N}(\mathrm{Tc}) \sim$ Light + Temperature, Ea $\sim$ Light, Eh $\sim$ Light + Temperature, Th $\sim$ Light * Temperature 276.1679

$\mathrm{N}(\mathrm{Tc}) \sim$ Light * Temperature, Ea Light, Eh Light + Temperature, Th Light * Temperature 276.3218

$\mathrm{N}(\mathrm{Tc}) \sim$ Light * Temperature, Ea Light, Eh $\sim$ Temperature, Th Light * Temperature 276.3254

$\mathrm{N}(\mathrm{Tc}) \sim$ Light * Temperature, Ea $\sim$ Light * Temperature, Eh $\sim$ Light * Temperature, Th Temperature 276.3706 $\mathrm{N}(\mathrm{Tc}) \sim$ Light + Temperature, Ea Light * Temperature, Eh Light * Temperature, Th Light 276.4026

$\mathrm{N}(\mathrm{Tc}) \sim$ Light, Ea $\sim$ Light, Eh $\sim$ Light + Temperature, Th Light 276.4123

$\mathrm{N}(\mathrm{Tc}) \sim$ Light * Temperature, Ea Temperature, Eh Temperature, Th Light 276.5106 
$\mathrm{N}(\mathrm{Tc}) \sim$ Light + Temperature, Ea $\sim 1$, Eh $\sim$ Light + Temperature, Th $\sim$ Light 276.5623

$\mathrm{N}(\mathrm{Tc}) \sim$ Light, Ea $\sim$ Temperature, Eh $\sim$ Temperature, Th Light 276.8301

$\mathrm{N}(\mathrm{Tc}) \sim$ Light $*$ Temperature, Ea $\sim$ Temperature, Eh $\sim 1$, Th $\sim$ Light 276.9847

$\mathrm{N}(\mathrm{Tc}) \sim$ Light * Temperature, Ea $\sim$ Light, Eh $\sim$ Light * Temperature, Th $\sim$ Light * Temperature 277.0157

N(Tc) Light + Temperature, Ea Light + Temperature, Eh Light, Th 1277.0865

$\mathrm{N}(\mathrm{Tc}) \sim$ Light * Temperature, Ea $\sim$ Light, Eh $\sim$ Light + Temperature, Th $\sim$ Light 277.1028

$\mathrm{N}(\mathrm{Tc}) \sim$ Light * Temperature, Ea Light * Temperature, Eh $\sim 1$, Th Light 277.1918

$\mathrm{N}(\mathrm{Tc}) \sim$ Light, Ea $\sim$ Light * Temperature, Eh $\sim$ Light, Th Light * Temperature 277.3807

$\mathrm{N}(\mathrm{Tc}) \sim$ Light * Temperature, Ea $\sim$ Light * Temperature, Eh $\sim$ Temperature, Th $\sim$ Light 277.4342

$\mathrm{N}(\mathrm{Tc}) \sim$ Light $*$ Temperature, Ea $\sim$ Light * Temperature, Eh $\sim$ Temperature, Th $\sim$ Light + Temperature 277.709

$\mathrm{N}(\mathrm{Tc}) \sim$ Light + Temperature, Ea $\sim$ Temperature, Eh 1, Th Light + Temperature 278.1267

$\mathrm{N}(\mathrm{Tc}) \sim$ Light, Ea $\sim 1$, Eh Light * Temperature, Th Light 278.1587

$\mathrm{N}(\mathrm{Tc}) \sim$ Light + Temperature, Ea $\sim$ Light + Temperature, Eh $\sim 1$, Th $\sim$ Light $*$ Temperature 278.2552

$\mathrm{N}(\mathrm{Tc}) \sim$ Light * Temperature, Ea Light + Temperature, Eh Light, Th $\sim 1278.2743$

$\mathrm{N}(\mathrm{Tc}) \sim$ Light, $\mathrm{Ea} \sim$ Light + Temperature, Eh $\sim$ Temperature, Th $\sim$ Light + Temperature 278.3031

$\mathrm{N}(\mathrm{Tc}) \sim$ Light * Temperature, Ea $\sim 1$, Eh $\sim$ Temperature, Th Light * Temperature 278.4601

$\mathrm{N}(\mathrm{Tc}) \sim$ Light * Temperature, Ea Light + Temperature, Eh Light * Temperature, Th Light 278.4977

$\mathrm{N}(\mathrm{Tc}) \sim$ Light, Ea $\sim$ Temperature, Eh $\sim 1$, Th Light + Temperature 278.5053

$\mathrm{N}(\mathrm{Tc}) \sim$ Light + Temperature, Ea $\sim 1$, Eh $\sim$ Light + Temperature, Th $\sim$ Light * Temperature 278.7089

$\mathrm{N}(\mathrm{Tc}) \sim$ Light + Temperature, Ea $\sim 1$, Eh $\sim$ Light * Temperature, Th $\sim$ Light * Temperature 278.8844

N(Tc) Light, Ea Temperature, Eh 1, Th Light 278.9943

$\mathrm{N}(\mathrm{Tc}) \sim$ Light + Temperature, Ea $\sim$ Light, Eh $\sim$ Light * Temperature, Th $\sim$ Light + Temperature 279.0238

$\mathrm{N}(\mathrm{Tc}) \sim$ Light + Temperature, Ea Light + Temperature, Eh $\sim$ Temperature, Th Light + Temperature 279.5183

$\mathrm{N}(\mathrm{Tc}) \sim$ Light, Ea $\sim$ Temperature, Eh $\sim$ Temperature, Th $\sim$ Light * Temperature 279.5668

$\mathrm{N}(\mathrm{Tc}) \sim$ Light + Temperature, Ea $\sim$ Light, Eh $\sim$ Light + Temperature, Th $\sim$ Temperature 279.5709

$\mathrm{N}(\mathrm{Tc}) \sim$ Light + Temperature, Ea $\sim 1$, Eh $\sim$ Light * Temperature, Th Light 279.7508

$\mathrm{N}(\mathrm{Tc}) \sim$ Light, Ea $\sim$ Light + Temperature, Eh $\sim$ Temperature, Th Light 279.7562 $\mathrm{N}(\mathrm{Tc}) \sim$ Light, Ea $\sim$ Light, Eh $\sim$ Light * Temperature, Th Light 279.8689 
$\mathrm{N}(\mathrm{Tc}) \sim$ Light + Temperature, Ea $\sim$ Light + Temperature, Eh $\sim$ Light, Th $\sim$ Temperature 280.3206

$\mathrm{N}(\mathrm{Tc}) \sim$ Light, Ea $\sim$ Light + Temperature, Eh $\sim$ Light * Temperature, Th $\sim$ Light * Temperature 280.3741

$\mathrm{N}(\mathrm{Tc}) \sim$ Light + Temperature, Ea $\sim$ Temperature, Eh $\sim$ Temperature, Th $\sim$ Light 280.5612

$\mathrm{N}(\mathrm{Tc}) \sim$ Light, Ea $\sim$ Light * Temperature, Eh 1, Th Light 280.5829

$\mathrm{N}(\mathrm{Tc}) \sim$ Light * Temperature, Ea $\sim 1$, Eh Light * Temperature, Th Light 280.8549

$\mathrm{N}(\mathrm{Tc}) \sim$ Light * Temperature, Ea $\sim$ Light, Eh $\sim$ Light, Th $\sim$ Light * Temperature 280.8566

$\mathrm{N}(\mathrm{Tc}) \sim$ Light + Temperature, Ea $\sim$ Light * Temperature, Eh $\sim 1, \mathrm{Th} \sim$ Light 281.3304

$\mathrm{N}(\mathrm{Tc}) \sim$ Light + Temperature, Ea $\sim$ Light, Eh $\sim$ Light * Temperature, Th Light 281.3797

$\mathrm{N}(\mathrm{Tc}) \sim$ Light * Temperature, Ea $\sim$ Light * Temperature, Eh $\sim$ Light, Th $\sim$ Light + Temperature 281.4434

$\mathrm{N}(\mathrm{Tc}) \sim$ Light, Ea $\sim$ Light * Temperature, Eh $\sim$ Temperature, Th Light 281.4559

$\mathrm{N}(\mathrm{Tc}) \sim$ Light, Ea $\sim$ Light * Temperature, Eh Light * Temperature, Th Light + Temperature 281.7418

$\mathrm{N}(\mathrm{Tc}) \sim$ Light, Ea $\sim$ Light + Temperature, Eh $\sim 1$, Th $\sim$ Light + Temperature 282.0866

N(Tc) Light * Temperature, Ea Light * Temperature, Eh 1, Th Light + Temperature 282.1048

$\mathrm{N}(\mathrm{Tc}) \sim$ Light $*$ Temperature, Ea $~ 1, \mathrm{Eh} \sim 1$, Th $\sim$ Light * Temperature 282.1176

$\mathrm{N}(\mathrm{Tc}) \sim$ Light $*$ Temperature, Ea $\sim$ Light + Temperature, Eh $\sim$ Light + Temperature, Th $\sim$ Light + Temperature 282.1793

$\mathrm{N}(\mathrm{Tc}) \sim$ Light * Temperature, Ea $\sim 1$, Eh $\sim$ Light * Temperature, Th $\sim$ Light + Temperature 282.1967

$\mathrm{N}(\mathrm{Tc}) \sim$ Light + Temperature, Ea $\sim$ Light, Eh $\sim$ Light * Temperature, Th $\sim$ Temperature 282.3424

$\mathrm{N}(\mathrm{Tc}) \sim$ Light, Ea $\sim$ Light + Temperature, Eh $\sim 1$, Th Light 282.3509

$\mathrm{N}(\mathrm{Tc}) \sim$ Light * Temperature, Ea $\sim$ Light, Eh $\sim$ Light + Temperature, Th $\sim$ Light + Temperature 282.4163

$\mathrm{N}(\mathrm{Tc}) \sim$ Light + Temperature, Ea $\sim$ Light, Eh $\sim$ Light + Temperature, Th Light + Temperature 282.4561

$\mathrm{N}(\mathrm{Tc}) \sim$ Light, Ea $\sim$ Light $*$ Temperature, Eh $\sim$ Temperature, Th $\sim$ Light + Temperature 282.4916

$\mathrm{N}(\mathrm{Tc}) \sim$ Light * Temperature, Ea $\sim$ Light, Eh $\sim 1$, Th $\sim$ Light * Temperature 282.5175

$\mathrm{N}(\mathrm{Tc}) \sim$ Light + Temperature, Ea Light * Temperature, Eh Light, Th 1282.5977

N(Tc) $\sim$ Light, Ea $\sim$ Light + Temperature, Eh Temperature, Th Light * Temperature 282.6596

$\mathrm{N}(\mathrm{Tc}) \sim$ Light $*$ Temperature, Ea $\sim$ Temperature, Eh $\sim$ Light + Temperature, Th $\sim$ Temperature 282.822

$\mathrm{N}(\mathrm{Tc}) \sim$ Light, Ea $\sim$ Temperature, Eh $\sim 1$, Th Light * Temperature 282.8223

$\mathrm{N}(\mathrm{Tc}) \sim$ Light * Temperature, Ea $\sim$ Light * Temperature, Eh Light, Th 1282.8308

$\mathrm{N}(\mathrm{Tc}) \sim$ Light * Temperature, Ea $\sim$ Light, Eh $\sim$ Light * Temperature, Th $\sim$ Light 282.885 
$\mathrm{N}(\mathrm{Tc}) \sim$ Light $*$ Temperature, Ea $\sim$ Light + Temperature, Eh $\sim$ Light, Th $\sim$ Temperature 282.9206 $\mathrm{N}(\mathrm{Tc}) \sim$ Light * Temperature, Ea $\sim 1$, Eh $\sim$ Light, Th Light * Temperature 282.9547 $\mathrm{N}(\mathrm{Tc}) \sim$ Light, Ea Light + Temperature, Eh Light, Th 1283.0761 $\mathrm{N}(\mathrm{Tc}) \sim$ Light, Ea $\sim$ Light * Temperature, Eh $\sim 1$, Th $\sim$ Light + Temperature 283.1735 $\mathrm{N}(\mathrm{Tc}) \sim$ Light, Ea $\sim$ Light + Temperature, Eh Light, Th Temperature 283.2188 $\mathrm{N}(\mathrm{Tc}) \sim$ Light, Ea $\sim 1$, Eh Light * Temperature, Th Light + Temperature 283.4524 $\mathrm{N}(\mathrm{Tc}) \sim$ Light + Temperature, Ea $\sim$ Light * Temperature, Eh $\sim$ Temperature, Th $\sim$ Light 283.5847 $\mathrm{N}(\mathrm{Tc}) \sim$ Light + Temperature, Ea $\sim$ Temperature, Eh $\sim 1$, Th $\sim$ Light * Temperature 283.6823 $\mathrm{N}(\mathrm{Tc}) \sim$ Light * Temperature, Ea $\sim$ Temperature, Eh Light + Temperature, Th 1284.0575 $\mathrm{N}(\mathrm{Tc}) \sim$ Light + Temperature, Ea $\sim$ Temperature, Eh Light + Temperature, Th 1284.8365 $\mathrm{N}(\mathrm{Tc}) \sim$ Light + Temperature, Ea Light * Temperature, Eh 1, Th Light * Temperature 284.8621

$\mathrm{N}(\mathrm{Tc}) \sim$ Light, Ea $\sim$ Light * Temperature, Eh $\sim$ Temperature, Th $\sim$ Light * Temperature 284.9095 $\mathrm{N}(\mathrm{Tc}) \sim$ Light, Ea $\sim$ Light * Temperature, Eh $\sim$ Light + Temperature, Th $\sim$ Light * Temperature 284.9744 $\mathrm{N}(\mathrm{Tc}) \sim$ Light, Ea $\sim$ Temperature, Eh Light + Temperature, Th 1285.0128

$\mathrm{N}(\mathrm{Tc}) \sim$ Light, Ea $\sim$ Light $*$ Temperature, Eh $\sim 1$, Th $\sim$ Light * Temperature 285.0789

$\mathrm{N}(\mathrm{Tc}) \sim$ Light + Temperature, Ea $\sim$ Light + Temperature, Eh $\sim 1$, Th $\sim$ Light + Temperature 285.2255

$\mathrm{N}(\mathrm{Tc}) \sim$ Light * Temperature, Ea $\sim$ Light, Eh $\sim$ Temperature, Th $\sim$ Light + Temperature 285.3402 $\mathrm{N}(\mathrm{Tc}) \sim$ Light + Temperature, Ea $\sim$ Light + Temperature, Eh $\sim$ 1, Th $\sim$ Light 285.3792 $\mathrm{N}(\mathrm{Tc}) \sim$ Light + Temperature, Ea $\sim$ Light + Temperature, Eh $\sim$ Light + Temperature, Th $\sim$ Light * Temperature 285.4485

$\mathrm{N}(\mathrm{Tc}) \sim$ Light * Temperature, Ea $\sim$ Light, Eh $\sim$ Light + Temperature, Th $\sim$ Temperature 285.5569 $\mathrm{N}(\mathrm{Tc}) \sim$ Light, Ea $\sim$ Light, Eh $\sim$ Light * Temperature, Th $\sim$ Light + Temperature 285.574

$\mathrm{N}(\mathrm{Tc}) \sim$ Light $*$ Temperature, Ea $\sim 1$, Eh $\sim$ Temperature, Th $\sim$ Light + Temperature 285.5937 $\mathrm{N}(\mathrm{Tc}) \sim$ Light + Temperature, Ea Light * Temperature, Eh 1, Th Light + Temperature 285.6004 $\mathrm{N}(\mathrm{Tc}) \sim$ Light + Temperature, Ea $\sim$ Temperature, Eh $\sim$ Light + Temperature, Th $\sim$ Temperature 286.119

$\mathrm{N}(\mathrm{Tc}) \sim$ Light + Temperature, Ea $\sim 1$, Eh $\sim$ Light + Temperature, Th $\sim$ Light + Temperature 286.6835 $\mathrm{N}(\mathrm{Tc}) \sim$ Light, Ea $\sim$ Temperature, Eh $\sim$ Light * Temperature, Th 1286.895

$\mathrm{N}(\mathrm{Tc}) \sim$ Light $*$ Temperature, Ea $\sim 1$, Eh $\sim$ Light + Temperature, Th $\sim$ Light + Temperature 287.8998 $\mathrm{N}(\mathrm{Tc}) \sim$ Light + Temperature, Ea $\sim$ Temperature, Eh $\sim$ Light * Temperature, Th $\sim 1288.3067$ $\mathrm{N}(\mathrm{Tc}) \sim$ Light, Ea $\sim$ Light * Temperature, Eh Light, Th $\sim 1288.3568$ 
$\mathrm{N}(\mathrm{Tc}) \sim$ Light $*$ Temperature, Ea $\sim$ Light * Temperature, Eh $\sim$ Light, Th $\sim$ Temperature 289.1704 $\mathrm{N}(\mathrm{Tc}) \sim$ Light $*$ Temperature, Ea $\sim$ Light, Eh $\sim$ Light * Temperature, Th $\sim$ Temperature 289.2669

$\mathrm{N}(\mathrm{Tc}) \sim$ Light * Temperature, Ea Temperature, Eh Light * Temperature, Th 1289.4587

$\mathrm{N}(\mathrm{Tc}) \sim$ Light, Ea $\sim$ Light + Temperature, Eh $\sim 1$, Th $\sim$ Light * Temperature 289.502

$\mathrm{N}(\mathrm{Tc}) \sim$ Light, Ea $\sim$ Temperature, Eh $\sim$ Light + Temperature, Th $\sim$ Temperature 289.511

$\mathrm{N}(\mathrm{Tc}) \sim$ Light, Ea Light, Eh Light + Temperature, Th Light * Temperature 289.7714

$\mathrm{N}(\mathrm{Tc}) \sim$ Light, Ea $\sim$ Light, Eh $\sim$ Light + Temperature, Th 1289.853

N(Tc) Light + Temperature, Ea $\sim$ Temperature, Eh Light * Temperature, Th $\sim$ Temperature 290.3937

$\mathrm{N}(\mathrm{Tc}) \sim$ Light, Ea $\sim 1$, Eh Light + Temperature, Th Light * Temperature 290.4795

$\mathrm{N}(\mathrm{Tc}) \sim$ Light + Temperature, Ea $\sim$ Light * Temperature, Eh $\sim$ Light, Th $\sim$ Light * Temperature 290.6325

$\mathrm{N}(\mathrm{Tc}) \sim$ Light + Temperature, Ea $\sim$ Light + Temperature, Eh $\sim$ Temperature, Th Light 291.0381

$\mathrm{N}(\mathrm{Tc}) \sim$ Light, Ea $\sim$ Temperature, Eh $\sim$ Light * Temperature, Th $\sim$ Temperature 291.955

$\mathrm{N}(\mathrm{Tc}) \sim$ Light * Temperature, Ea $\sim 1$, Eh $\sim$ Light, Th $\sim$ Light + Temperature 292.1573

$\mathrm{N}(\mathrm{Tc}) \sim$ Light * Temperature, Ea Temperature, Eh Light, Th 1292.5478

$\mathrm{N}(\mathrm{Tc}) \sim$ Light + Temperature, Ea $\sim 1$, Eh Light + Temperature, Th Temperature 292.6852

$\mathrm{N}(\mathrm{Tc}) \sim$ Light, Ea $\sim 1$, Eh Light, Th Light 292.8407

$\mathrm{N}(\mathrm{Tc}) \sim$ Light + Temperature, Ea $\sim 1$, Eh $\sim$ Light * Temperature, Th $\sim$ Temperature 292.9205

$\mathrm{N}(\mathrm{Tc}) \sim$ Light * Temperature, Ea $\sim 1$, Eh Light, Th Light 292.9209

$\mathrm{N}(\mathrm{Tc}) \sim$ Light $*$ Temperature, Ea $\sim 1$, Eh $\sim 1$, Th $\sim$ Light + Temperature 293.0151

$\mathrm{N}(\mathrm{Tc}) \sim$ Light, Ea $\sim 1$, Eh Light * Temperature, Th Light * Temperature 293.0645 $\mathrm{N}(\mathrm{Tc}) \sim$ Light + Temperature, Ea $\sim 1$, Eh Light, Th Light 293.3929

$\mathrm{N}(\mathrm{Tc}) \sim$ Light, Ea $\sim$ Light, Eh $\sim$ Light, Th Light 293.9392

$\mathrm{N}(\mathrm{Tc}) \sim$ Light + Temperature, Ea $\sim$ Light, Eh $\sim$ Light, Th Light 294.3765

$\mathrm{N}(\mathrm{Tc}) \sim$ Light * Temperature, Ea $\sim$ Light, Eh $\sim$ Light, Th Light 294.4514

$\mathrm{N}(\mathrm{Tc}) \sim$ Light * Temperature, Ea $\sim$ Light, Eh $\sim 1$, Th $\sim$ Light + Temperature 294.5006

$\mathrm{N}(\mathrm{Tc}) \sim$ Light, Ea $\sim$ Light, Eh $\sim$ Light * Temperature, Th 1294.5283

$\mathrm{N}(\mathrm{Tc}) \sim$ Light, Ea $\sim$ Light, Eh $\sim$ Light * Temperature, Th Light * Temperature 294.9715

$\mathrm{N}(\mathrm{Tc}) \sim$ Light, Ea $\sim$ Light, Eh $\sim$ Light + Temperature, Th $\sim$ Temperature 295.1146

$\mathrm{N}(\mathrm{Tc}) \sim$ Light * Temperature, Ea $\sim$ Light, Eh $\sim$ Light, Th Light + Temperature 295.3686 
$\mathrm{N}(\mathrm{Tc}) \sim$ Light + Temperature, Ea Light, Eh Light + Temperature, Th 1296.1067 $\mathrm{N}(\mathrm{Tc}) \sim$ Light * Temperature, Ea Light, Eh 1, Th Light 296.1609 $\mathrm{N}(\mathrm{Tc}) \sim$ Light + Temperature, Ea Light * Temperature, Eh $\sim$ Light + Temperature, Th $\sim$ Light + Temperature 296.2356 $\mathrm{N}(\mathrm{Tc}) \sim$ Light + Temperature, Ea Light + Temperature, Eh $\sim$ Light * Temperature, Th $\sim$ Light + Temperature 296.3661 $\mathrm{N}(\mathrm{Tc}) \sim$ Light, Ea $\sim$ Light, Eh $\sim$ Light + Temperature, Th $\sim$ Light + Temperature 296.7377 $\mathrm{N}(\mathrm{Tc}) \sim 1$, Ea Temperature, Eh Light + Temperature, Th Light + Temperature 296.808 $\mathrm{N}(\mathrm{Tc}) \sim$ Light * Temperature, Ea 1, Eh Light * Temperature, Th Temperature 297.1076 $\mathrm{N}(\mathrm{Tc}) \sim$ Light * Temperature, Ea $\sim$ Temperature, Eh $\sim$ Light, Th $\sim$ Temperature 297.7415 $\mathrm{N}(\mathrm{Tc}) \sim$ Light, Ea 1, Eh Light, Th Light + Temperature 298.0993

$\mathrm{N}(\mathrm{Tc}) \sim$ Light, Ea $\sim$ Light, Eh Light * Temperature, Th Temperature 298.2826 $\mathrm{N}(\mathrm{Tc}) \sim$ Light + Temperature, Ea Light, Eh $\sim$ Light * Temperature, Th Light * Temperature 298.5075 $\mathrm{N}(\mathrm{Tc}) \sim$ Light + Temperature, Ea $\sim$ Light, Eh $\sim$ Temperature, Th Light * Temperature 298.894 $\mathrm{N}(\mathrm{Tc}) \sim 1$, Ea $\sim$ Light + Temperature, Eh $\sim$ Light + Temperature, Th $\sim$ Light + Temperature 298.924 $\mathrm{N}(\mathrm{Tc}) \sim$ Light + Temperature, Ea $\sim$ Light, Eh $\sim$ Light, Th $\sim$ Light * Temperature 299.2831 $\mathrm{N}(\mathrm{Tc}) \sim$ Light, Ea Light, Eh Light, Th Light + Temperature 299.4619 $\mathrm{N}(\mathrm{Tc}) \sim$ Light + Temperature, Ea $\sim$ Temperature, Eh $\sim$ Temperature, Th Light * Temperature 299.5956 $\mathrm{N}(\mathrm{Tc}) \sim$ Light + Temperature, Ea $\sim$ 1, Eh $\sim$ Light, Th Light + Temperature 299.689 $\mathrm{N}(\mathrm{Tc}) \sim 1$, Ea Temperature, Eh Light + Temperature, Th Light 299.9935

$\mathrm{N}(\mathrm{Tc}) \sim$ Light * Temperature, Ea $\sim$ Light, Eh $\sim$ Temperature, Th Light 300.4103 $\mathrm{N}(\mathrm{Tc}) \sim$ Light + Temperature, Ea 1, Eh $\sim$ Temperature, Th Light * Temperature 300.5577 $\mathrm{N}(\mathrm{Tc}) \sim$ Light + Temperature, Ea Light, Eh Light, Th Light + Temperature 300.7192 $\mathrm{N}(\mathrm{Tc}) \sim 1$, Ea $\sim$ Light + Temperature, Eh Light + Temperature, Th Light 301.0251 $\mathrm{N}(\mathrm{Tc}) \sim$ Light + Temperature, Ea $\sim 1$, Eh $\sim$ Light, Th $\sim$ Light * Temperature 301.0812 $\mathrm{N}(\mathrm{Tc}) \sim$ Light + Temperature, Ea $\sim 1$, Eh $\sim$ Light + Temperature, Th 1301.2249 $\mathrm{N}(\mathrm{Tc}) \sim$ Light + Temperature, Ea $\sim$ Light, Eh $\sim$ Temperature, Th $\sim$ Light + Temperature 301.2695 $\mathrm{N}(\mathrm{Tc}) \sim$ Light + Temperature, Ea $\sim$ Light, Eh $\sim$ Light, Th $\sim$ Temperature 301.3428 $\mathrm{N}(\mathrm{Tc}) \sim 1$, Ea $\sim$ Temperature, Eh $\sim$ Light * Temperature, Th Light + Temperature 301.483 $\mathrm{N}(\mathrm{Tc}) \sim 1$, Ea $\sim$ Light * Temperature, Eh Light + Temperature, Th Light 301.7176 $\mathrm{N}(\mathrm{Tc}) \sim$ Light + Temperature, Ea 1, Eh $\sim$ Temperature, Th Light + Temperature 302.0505 
$\mathrm{N}(\mathrm{Tc}) \sim$ Light * Temperature, Ea Light, Eh Light + Temperature, Th 1302.1916

$\mathrm{N}(\mathrm{Tc}) \sim$ Light + Temperature, Ea Light, Eh Light * Temperature, Th 1302.5611

$\mathrm{N}(\mathrm{Tc}) \sim$ Light + Temperature, Ea $\sim$ Light * Temperature, Eh $\sim$ Temperature, Th $\sim$ Light + Temperature 302.631 $\mathrm{N}(\mathrm{Tc}) \sim$ Temperature, Ea $\sim$ Temperature, Eh $\sim$ Light + Temperature, Th $\sim$ Light + Temperature 302.8096 $\mathrm{N}(\mathrm{Tc}) \sim$ Light + Temperature, Ea $\sim$ Light + Temperature, Eh $\sim$ Temperature, Th $\sim$ Light * Temperature 303.0649 $\mathrm{N}(\mathrm{Tc}) \sim$ Light + Temperature, Ea Temperature, Eh Light, Th 1303.1928

$\mathrm{N}(\mathrm{Tc}) \sim$ Light, Ea $\sim 1$, Eh $\sim$ Light + Temperature, Th $\sim$ Light + Temperature 303.9737 $\mathrm{N}(\mathrm{Tc}) \sim 1$, Ea $\sim$ Light + Temperature, Eh $\sim$ Light * Temperature, Th Light + Temperature 303.9876 $\mathrm{N}(\mathrm{Tc}) \sim 1$, Ea Light * Temperature, Eh Light + Temperature, Th Light + Temperature 304.0134 $\mathrm{N}(\mathrm{Tc}) \sim$ Light, Ea 1, Eh Light + Temperature, Th 1304.0941 $\mathrm{N}(\mathrm{Tc}) \sim$ Light * Temperature, Ea 1, Eh 1, Th Light 304.113

$\mathrm{N}(\mathrm{Tc}) \sim 1$, Ea $\sim$ Temperature, Eh Light, Th Light * Temperature 304.1221 $\mathrm{N}(\mathrm{Tc}) \sim 1$, Ea Temperature, Eh Light * Temperature, Th Light * Temperature 304.1259 $\mathrm{N}(\mathrm{Tc}) \sim$ Light * Temperature, Ea Light + Temperature, Eh $\sim 1$, Th $\sim 1 \quad 304.151$ $\mathrm{N}(\mathrm{Tc}) \sim$ Light, Ea 1, Eh 1, Th Light 304.344

$\mathrm{N}(\mathrm{Tc}) \sim$ Light * Temperature, Ea $\sim 1$, Eh $\sim$ Temperature, Th Light 304.5717 $\mathrm{N}(\mathrm{Tc}) \sim$ Light, Ea $\sim 1$, Eh $\sim$ Temperature, Th Light 304.6679

$\mathrm{N}(\mathrm{Tc}) \sim 1$, Ea $\sim$ Temperature, Eh Light + Temperature, Th Light* Temperature 304.8585 $\mathrm{N}(\mathrm{Tc}) \sim$ Light * Temperature, Ea $\sim$ Light, Eh $\sim$ Light * Temperature, Th $\sim$ Light + Temperature 305.1643 $\mathrm{N}(\mathrm{Tc}) \sim$ Temperature, Ea $\sim$ Light + Temperature, Eh Light + Temperature, Th Light + Temperature 305.1835 $\mathrm{N}(\mathrm{Tc}) \sim$ Light * Temperature, Ea Temperature, Eh 1, Th 1305.6676

$\mathrm{N}(\mathrm{Tc}) \sim$ Temperature, Ea $\sim$ Temperature, Eh $\sim$ Light + Temperature, Th Light 305.8742 $\mathrm{N}(\mathrm{Tc}) \sim$ Light + Temperature, Ea 1, Eh 1, Th Light 305.9489

$\mathrm{N}(\mathrm{Tc}) \sim$ Light, Ea $\sim 1$, Eh $\sim$ Light + Temperature, Th $\sim$ Temperature 306.4161 $\mathrm{N}(\mathrm{Tc}) \sim$ Temperature, Ea $\sim$ Light + Temperature, Eh $\sim$ Light + Temperature, Th $\sim$ Light 307.0733 N(Tc) Light * Temperature, Ea Light, Eh Light, Th 1307.2029 $\mathrm{N}(\mathrm{Tc}) \sim$ Light + Temperature, Ea $\sim 1$, Eh $\sim$ Temperature, Th $\sim$ Light 307.2111 $\mathrm{N}(\mathrm{Tc}) \sim$ Light, Ea Light, Eh 1, Th Light 307.2442 $\mathrm{N}(\mathrm{Tc}) \sim$ Light, Ea Light, Eh Light, Th Light * Temperature 307.3082 
$\mathrm{N}(\mathrm{Tc}) \sim 1$, Ea Light + Temperature, Eh Light * Temperature, Th Light * Temperature 307.5703 $\mathrm{N}(\mathrm{Tc}) \sim$ Light, Ea $\sim$ Light, Eh $\sim$ Temperature, Th Light * Temperature 307.634 $\mathrm{N}(\mathrm{Tc}) \sim 1$, Ea Light + Temperature, Eh Light, Th Light * Temperature 307.662 $\mathrm{N}(\mathrm{Tc}) \sim$ Light + Temperature, Ea Light, Eh Light, Th 1307.6916 $\mathrm{N}(\mathrm{Tc}) \sim$ Light, Ea $\sim$ Light, Eh $\sim$ Temperature, Th $\sim$ Light 307.7491

$\mathrm{N}(\mathrm{Tc}) \sim$ Light + Temperature, Ea 1, Eh 1, Th Light * Temperature 307.8049 $\mathrm{N}(\mathrm{Tc}) \sim$ Temperature, Ea $\sim$ Temperature, Eh $\sim$ Light * Temperature, Th $\sim$ Light + Temperature 307.9282 $\mathrm{N}(\mathrm{Tc}) \sim 1$, Ea $\sim$ Light + Temperature, Eh $\sim$ Light + Temperature, Th Light * Temperature 308.0333 $\mathrm{N}(\mathrm{Tc}) \sim$ Temperature, Ea $\sim$ Light * Temperature, Eh $\sim$ Light + Temperature, Th $\sim$ Light 308.1153 $\mathrm{N}(\mathrm{Tc}) \sim 1$, Ea Light * Temperature, Eh Light * Temperature, Th Light + Temperature 308.2389 $\mathrm{N}(\mathrm{Tc}) \sim 1$, Ea $\sim$ Light + Temperature, Eh $\sim$ Light * Temperature, Th Light 308.3271 $\mathrm{N}(\mathrm{Tc}) \sim$ Light * Temperature, Ea Light, Eh Light * Temperature, Th 1308.8908 $\mathrm{N}(\mathrm{Tc}) \sim 1$, Ea $\sim$ Light * Temperature, Eh Light + Temperature, Th Light * Temperature 308.9908 $\mathrm{N}(\mathrm{Tc}) \sim$ Light + Temperature, Ea Light, Eh 1, Th Light 309.0295 $\mathrm{N}(\mathrm{Tc}) \sim$ Light, Ea Light, Eh Light, Th 1309.1829

$\mathrm{N}(\mathrm{Tc}) \sim$ Temperature, Ea $\sim$ Light, Eh $\sim$ Light * Temperature, Th $\sim$ Light + Temperature 309.1851 $\mathrm{N}(\mathrm{Tc}) \sim$ Light, Ea 1, Eh Temperature, Th Light * Temperature 309.3632 $\mathrm{N}(\mathrm{Tc}) \sim$ Light, Ea Temperature, Eh Light, Th Temperature 309.4907 $\mathrm{N}(\mathrm{Tc}) \sim$ Temperature, Ea $\sim$ Temperature, Eh $\sim$ Light, Th $\sim$ Light * Temperature 309.5728 $\mathrm{N}(\mathrm{Tc}) \sim$ Light, Ea 1, Eh Temperature, Th Light + Temperature 309.5776 $\mathrm{N}(\mathrm{Tc}) \sim$ Light, Ea $\sim 1$, Eh 1, Th Light + Temperature 309.5779 $\mathrm{N}(\mathrm{Tc}) \sim$ Light * Temperature, Ea Light * Temperature, Eh 1, Th 1309.6206 $\mathrm{N}(\mathrm{Tc}) \sim$ Light + Temperature, Ea $\sim$ Light, Eh $\sim 1$, Th $\sim$ Light + Temperature 309.9199 $\mathrm{N}(\mathrm{Tc}) \sim 1$, Ea $\sim$ Light + Temperature, Eh 1, Th Light * Temperature 309.9569 $\mathrm{N}(\mathrm{Tc}) \sim$ Light * Temperature, Ea Light + Temperature, Eh $\sim 1$, Th $\sim$ Temperature 309.9882 $\mathrm{N}(\mathrm{Tc}) \sim$ Light, Ea 1, Eh Light, Th Light * Temperature 310.1579 $\mathrm{N}(\mathrm{Tc}) \sim$ Light * Temperature, Ea $\sim$ Temperature, Eh $\sim 1$, Th $\sim$ Temperature 310.1735 $\mathrm{N}(\mathrm{Tc}) \sim$ Light, Ea $\sim 1$, Eh $\sim$ Light * Temperature, Th Temperature 310.2506 $\mathrm{N}(\mathrm{Tc}) \sim 1$, Ea Light * Temperature, Eh Light * Temperature, Th Light 310.4281 

$\mathrm{N}(\mathrm{Tc}) \sim$ Light + Temperature, Ea $\sim$ Light + Temperature, Eh $\sim 1$, Th $\sim$ Temperature 310.8883

$\mathrm{N}(\mathrm{Tc}) \sim$ Light, Ea Light, Eh 1, Th Light * Temperature 311.441 $\mathrm{N}(\mathrm{Tc}) \sim$ Light, Ea $\sim$ Light, Eh $\sim$ Light, Th $\sim$ Temperature 312.2865

N(Tc) Light, Ea Temperature, Eh Light, Th 1312.5368

$\mathrm{N}(\mathrm{Tc}) \sim$ Temperature, Ea $\sim$ Light + Temperature, Eh $\sim$ Light, Th $\sim$ Light $*$ Temperature 312.7992 $\mathrm{N}(\mathrm{Tc}) \sim$ Light + Temperature, Ea $\sim$ Light, Eh $\sim 1$, Th $\sim$ Light * Temperature 312.961 $\mathrm{N}(\mathrm{Tc}) \sim 1$, Ea $\sim$ Light * Temperature, Eh $\sim$ Light, Th $\sim$ Light * Temperature $\quad 313.125$

$\mathrm{N}(\mathrm{Tc}) \sim$ Light, Ea $~ 1, \mathrm{Eh} \sim$ Light * Temperature, Th $\sim 1313.7253$

$\mathrm{N}(\mathrm{Tc}) \sim$ Light $*$ Temperature, Ea $\sim$ Temperature, Eh $\sim$ Light * Temperature, Th $\sim$ Temperature 313.8653 $\mathrm{N}(\mathrm{Tc}) \sim$ Light, Ea $\sim$ Light, Eh $\sim 1$, Th $\sim$ Light + Temperature 313.9179

$\mathrm{N}(\mathrm{Tc}) \sim$ Temperature, Ea $\sim$ Light + Temperature, Eh $\sim$ Light * Temperature, Th $\sim$ Light 314.6856 $\mathrm{N}(\mathrm{Tc}) \sim$ Light + Temperature, Ea 1, Eh Light * Temperature, Th 1314.7322

$\mathrm{N}(\mathrm{Tc}) \sim$ Temperature, Ea $\sim$ Light + Temperature, Eh $\sim$ Light + Temperature, Th $\sim$ Light * Temperature 314.77 $\mathrm{N}(\mathrm{Tc}) \sim$ Light * Temperature, Ea $\sim$ Light + Temperature, Eh $\sim$ Temperature, Th $\sim$ Temperature 314.8275 $\mathrm{N}(\mathrm{Tc}) \sim$ Light + Temperature, Ea $\sim 1$, Eh $\sim 1$, Th $\sim$ Light + Temperature 314.8564 $\mathrm{N}(\mathrm{Tc}) \sim$ Light, Ea $\sim$ Light, Eh $\sim$ Temperature, Th Light + Temperature 314.9768 $\mathrm{N}(\mathrm{Tc}) \sim 1$, Ea $\sim$ Light * Temperature, Eh $\sim 1$, Th $\sim$ Light * Temperature 315.0966 $\mathrm{N}(\mathrm{Tc}) \sim 1$, Ea $\sim$ Light + Temperature, Eh $\sim$ Temperature, Th $\sim$ Light * Temperature 315.1511 $\mathrm{N}(\mathrm{Tc}) \sim$ Temperature, Ea $\sim$ Light + Temperature, Eh $\sim 1$, Th $\sim$ Light * Temperature 315.3067 $\mathrm{N}(\mathrm{Tc}) \sim$ Light * Temperature, Ea $\sim$ Temperature, Eh $\sim$ Temperature, Th $\sim 1315.3411$ $\mathrm{N}(\mathrm{Tc}) \sim 1$, Ea $\sim$ Temperature, Eh $\sim 1$, Th $\sim$ Light * Temperature 315.6076 $\mathrm{N}(\mathrm{Tc}) \sim$ Light $*$ Temperature, Ea $\sim 1$, Eh $\sim$ Light + Temperature, Th $\sim 1315.8157$ $\mathrm{N}(\mathrm{Tc}) \sim$ Light * Temperature, Ea $\sim$ Light * Temperature, Eh $\sim 1, \mathrm{Th} \sim$ Temperature 315.8919 
$\mathrm{N}(\mathrm{Tc}) \sim$ Light + Temperature, Ea $\sim$ Temperature, Eh $\sim$ Light, Th $\sim$ Temperature 315.9919 
$\mathrm{N}(\mathrm{Tc}) \sim$ Light + Temperature, Ea Temperature, Eh 1, Th 1322.4819

$N(T c) \sim T$ Temperature, Ea $\sim$ Temperature, Eh $\sim$ light, Th $\sim$ Light

$\mathrm{N}(\mathrm{Tc}) \sim$ Temperature, Ea $\sim$ Temperature, Eh $\sim$ Light, Th Light + Temperature 322.9959

$\mathrm{N}(\mathrm{Tc}) \sim 1$, Ea Light * Temperature, Eh Light, Th Light 323.2074

$\mathrm{N}(\mathrm{Tc}) \sim 1$, Ea Light + Temperature, Eh Temperature, Th Light 324.0626

$\mathrm{N}(\mathrm{Tc}) \sim$ Light, Ea $\sim$ Light * Temperature, Eh 1, Th 1324.1169

$\mathrm{N}(\mathrm{Tc}) \sim$ Light * Temperature, Ea Light, Eh Light, Th Temperature 324.1908

$\mathrm{N}(\mathrm{Tc}) \sim$ Temperature, Ea $\sim$ Light + Temperature, Eh 1, Th Light 324.2105

$\mathrm{N}(\mathrm{Tc}) \sim$ Light + Temperature, Ea $\sim$ Light * Temperature, Eh $\sim$ Light, Th $\sim$ Temperature 324.2339

$\mathrm{N}(\mathrm{Tc}) \sim 1$, Ea $\sim$ Temperature, Eh 1, Th Light + Temperature 324.448

$\mathrm{N}(\mathrm{Tc}) \sim$ Light, Ea Light + Temperature, Eh 1, Th 1324.5832

$\mathrm{N}(\mathrm{Tc}) \sim 1$, Ea $\sim$ Light + Temperature, Eh $\sim$ Temperature, Th $\sim$ Light + Temperature 324.588

$\mathrm{N}(\mathrm{Tc}) \sim$ Temperature, Ea Light + Temperature, Eh $\sim$ Light, Th $\sim$ Light 325.0398 $\mathrm{N}(\mathrm{Tc}) \sim$ Light * Temperature, Ea 1, Eh 1, Th 1325.5148

$\mathrm{N}(\mathrm{Tc}) \sim$ Temperature, Ea Light + Temperature, Eh 1, Th Light + Temperature 325.5307

$\mathrm{N}(\mathrm{Tc}) \sim$ Temperature, Ea $\sim$ Temperature, Eh Light * Temperature, Th Light * Temperature 325.5882 $\mathrm{N}(\mathrm{Tc}) \sim$ Temperature, Ea Temperature, Eh Temperature, Th Light * Temperature 325.8044

$\mathrm{N}(\mathrm{Tc}) \sim$ Temperature, Ea Light + Temperature, Eh Light, Th Light + Temperature 325.8119

$\mathrm{N}(\mathrm{Tc}) \sim$ Light * Temperature, Ea 1, Eh Temperature, Th 1325.8322

$\mathrm{N}(\mathrm{Tc}) \sim$ Light, Ea $\sim$ Temperature, Eh 1, Th Temperature 326.1391

$\mathrm{N}(\mathrm{Tc}) \sim$ Light * Temperature, Ea $\sim 1$, Eh $\sim$ Light, Th Temperature 326.6903

$\mathrm{N}(\mathrm{Tc}) \sim$ Temperature, Ea Light * Temperature, Eh Light * Temperature, Th Light * Temperature 326.8345

$\mathrm{N}(\mathrm{Tc}) \sim$ Light + Temperature, Ea Light + Temperature, Eh Temperature, Th 1326.8511

$\mathrm{N}(\mathrm{Tc}) \sim$ Light * Temperature, Ea $\sim$ Light * Temperature, Eh $\sim$ Temperature, Th $\sim$ Temperature 327.1159

$\mathrm{N}(\mathrm{Tc}) \sim 1$, Ea $\sim$ Temperature, Eh $\sim$ Temperature, Th Light + Temperature 327.57

$\mathrm{N}(\mathrm{Tc}) \sim$ Light + Temperature, Ea Temperature, Eh 1, Th Temperature 327.6146

$\mathrm{N}(\mathrm{Tc}) \sim 1$, Ea $\sim 1$, Eh $\sim$ Light + Temperature, Th $\sim$ Light * Temperature 327.6788 $\mathrm{N}(\mathrm{Tc}) \sim$ Light * Temperature, Ea Light, Eh 1, Th 1327.7448 


365
476
536
62
114
513
357
304
337
233
339
112
109
503
104
45
40
538
236
52
17
270
486
528
25
211
42
586
93

$\mathrm{N}(\mathrm{Tc}) \sim$ Temperature, Ea Light * Temperature, Eh Temperature, Th Light * Temperature 327.8011

$\mathrm{N}(\mathrm{Tc}) \sim$ Light + Temperature, Ea $\sim$ Light * Temperature, Eh $\sim 1$, Th $\sim 1328.0396$

$\mathrm{N}(\mathrm{Tc}) \sim$ Light * Temperature, Ea Light, Eh Temperature, Th 1328.087

$\mathrm{N}(\mathrm{Tc}) \sim 1$, Ea $\sim$ Temperature, Eh $\sim$ Temperature, Th Light 328.4985

$\mathrm{N}(\mathrm{Tc}) \sim 1$, Ea Light * Temperature, Eh Temperature, Th Light + Temperature 328.77

$\mathrm{N}(\mathrm{Tc}) \sim$ Light * Temperature, Ea 1, Eh $\sim$ Temperature, Th Temperature 328.8338

$\mathrm{N}(\mathrm{Tc}) \sim$ Temperature, Ea Light * Temperature, Eh Light, Th Light 329.1763

$\mathrm{N}(\mathrm{Tc}) \sim$ Temperature, Ea $\sim$ Temperature, Eh 1, Th Light + Temperature 329.2685

$\mathrm{N}(\mathrm{Tc}) \sim$ Temperature, Ea $\sim$ Light + Temperature, Eh Temperature, Th Light 329.3902

$\mathrm{N}(\mathrm{Tc}) \sim$ Light, Ea $\sim$ Light * Temperature, Eh $\sim$ Light, Th Temperature 329.5589

$\mathrm{N}(\mathrm{Tc}) \sim$ Temperature, Ea $\sim$ Light + Temperature, Eh Temperature, Th Light + Temperature 329.8563

$\mathrm{N}(\mathrm{Tc}) \sim 1$, Ea Light * Temperature, Eh Temperature, Th Light 329.8854

$\mathrm{N}(\mathrm{Tc}) \sim 1$, Ea $\sim$ Light * Temperature, Eh Light, Th Light + Temperature 329.9142

$\mathrm{N}(\mathrm{Tc}) \sim$ Light * Temperature, Ea 1, Eh $\sim 1$, Th $\sim$ Temperature 330.3974

$\mathrm{N}(\mathrm{Tc}) \sim 1$, Ea Light * Temperature, Eh $\sim 1$, Th Light + Temperature 330.6093

$\mathrm{N}(\mathrm{Tc}) \sim 1$, Ea $\sim$ Light, Eh $\sim$ Light + Temperature, Th $\sim$ Light * Temperature 330.6691 $\mathrm{N}(\mathrm{Tc}) \sim 1$, Ea $\sim$ Light, Eh $\sim$ Temperature, Th Light * Temperature 331.2031

$\mathrm{N}(\mathrm{Tc}) \sim$ Light * Temperature, Ea Light, Eh $\sim$ Temperature, Th Temperature 331.4548

$\mathrm{N}(\mathrm{Tc}) \sim$ Light, Ea $\sim$ Light * Temperature, Eh $\sim$ Temperature, Th $\sim 1331.8476$

$\mathrm{N}(\mathrm{Tc}) \sim 1$, Ea $\sim$ Temperature, Eh $~ 1$, Th $\sim$ Light 331.9452

$\mathrm{N}(\mathrm{Tc}) \sim 1$, Ea 1, Eh Light + Temperature, Th Light 332.0933

$\mathrm{N}(\mathrm{Tc}) \sim$ Temperature, Ea $\sim 1$, Eh $\sim$ Light + Temperature, Th $\sim$ Light * Temperature 332.38

$\mathrm{N}(\mathrm{Tc}) \sim$ Light + Temperature, Ea Light * Temperature, Eh Temperature, Th 1332.6862

$\mathrm{N}(\mathrm{Tc}) \sim$ Light * Temperature, Ea $\sim$ Light, Eh $\sim 1$, Th Temperature 332.8514

$\mathrm{N}(\mathrm{Tc}) \sim 1$, Ea $\sim 1$, Eh $\sim$ Light * Temperature, Th Light * Temperature 333.3909

$\mathrm{N}(\mathrm{Tc}) \sim$ Light, Ea $\sim$ Light + Temperature, Eh $\sim$ Temperature, Th $\sim 1333.4885$

$\mathrm{N}(\mathrm{Tc}) \sim 1$, Ea Light, Eh Light + Temperature, Th Light 333.5272

$\mathrm{N}(\mathrm{Tc}) \sim$ Light * Temperature, Ea Light + Temperature, Eh Temperature, Th 1333.9138

$\mathrm{N}(\mathrm{Tc}) \sim 1$, Ea $\sim$ Light + Temperature, Eh $\sim$ Light + Temperature, Th $\sim$ Temperature 333.975 
$\mathrm{N}(\mathrm{Tc}) \sim$ Temperature, Ea $\sim$ Light * Temperature, Eh $\sim$ Temperature, Th Light + Temperature 334.1117

$\mathrm{N}(\mathrm{Tc}) \sim$ Temperature, Ea $\sim$ Temperature, Eh $\sim$ Temperature, Th Light 334.1773

$\mathrm{N}(\mathrm{Tc}) \sim$ Temperature, Ea $\sim$ Light, Eh $\sim$ Temperature, Th Light * Temperature 334.2243

$\mathrm{N}(\mathrm{Tc}) \sim 1$, Ea Light * Temperature, Eh 1, Th Light 334.2862

$\mathrm{N}(\mathrm{Tc}) \sim 1$, Ea $\sim$ Light + Temperature, Eh Light * Temperature, Th $\sim 1 \quad 334.387$

$\mathrm{N}(\mathrm{Tc}) \sim 1$, Ea $\sim$ Light, Eh $\sim$ Light * Temperature, Th $\sim$ Light + Temperature 334.7392

$\mathrm{N}(\mathrm{Tc}) \sim$ Temperature, Ea $\sim$ Light * Temperature, Eh $\sim$ Light, Th $\sim$ Light + Temperature 335.2161

$\mathrm{N}(\mathrm{Tc}) \sim 1$, Ea 1, Eh Temperature, Th Light * Temperature 335.3371

$\mathrm{N}(\mathrm{Tc}) \sim 1$, Ea Light + Temperature, Eh Light + Temperature, Th 1335.4233

$\mathrm{N}(\mathrm{Tc}) \sim 1$, Ea 1, Eh Light * Temperature, Th Light 335.4764

$\mathrm{N}(\mathrm{Tc}) \sim$ Temperature, Ea $\sim$ Light * Temperature, Eh $\sim$ Temperature, Th Light 335.5824

$\mathrm{N}(\mathrm{Tc}) \sim$ Temperature, Ea $\sim$ Light, Eh Light + Temperature, Th Light * Temperature 335.6227

$\mathrm{N}(\mathrm{Tc}) \sim$ Temperature, Ea Light * Temperature, Eh 1, Th Light + Temperature 335.6453

$\mathrm{N}(\mathrm{Tc}) \sim$ Light + Temperature, Ea $\sim$ Light + Temperature, Eh $\sim$ Temperature, Th $\sim$ Temperature 335.8486

$\mathrm{N}(\mathrm{Tc}) \sim$ Temperature, Ea Temperature, Eh Temperature, Th Light + Temperature 336.0578

$\mathrm{N}(\mathrm{Tc}) \sim$ Light, Ea $\sim$ Light + Temperature, Eh $\sim$ Temperature, Th $\sim$ Temperature 336.2749

$\mathrm{N}(\mathrm{Tc}) \sim 1$, Ea 1, Eh Light, Th Light * Temperature 336.3286

$\mathrm{N}(\mathrm{Tc}) \sim 1$, Ea 1, Eh Light + Temperature, Th Light + Temperature 336.4434

$\mathrm{N}(\mathrm{Tc}) \sim 1$, Ea $\sim$ Light, Eh $\sim$ Light * Temperature, Th $\sim$ Light * Temperature 336.7489

$\mathrm{N}(\mathrm{Tc}) \sim$ Light + Temperature, Ea $\sim$ Light, Eh $\sim$ Temperature, Th $\sim$ Temperature 336.7555

$\mathrm{N}(\mathrm{Tc}) \sim 1$, Ea Temperature, Eh Light + Temperature, Th 1337.3255

$\mathrm{N}(\mathrm{Tc}) \sim$ Temperature, Ea $\sim$ Temperature, Eh $\sim 1$, Th Light 337.3288

$\mathrm{N}(\mathrm{Tc}) \sim$ Temperature, Ea 1, Eh Light + Temperature, Th Light 337.6315

$\mathrm{N}(\mathrm{Tc}) \sim$ Light * Temperature, Ea Light * Temperature, Eh Temperature, Th 1337.6768

$\mathrm{N}(\mathrm{Tc}) \sim$ Light, Ea $\sim$ Light * Temperature, Eh $\sim$ Temperature, Th $\sim$ Temperature 337.6995

$\mathrm{N}(\mathrm{Tc}) \sim 1$, Ea Light, Eh Light * Temperature, Th Light 337.8195

$\mathrm{N}(\mathrm{Tc}) \sim$ Temperature, Ea $\sim 1$, Eh $\sim$ Light * Temperature, Th $\sim$ Light * Temperature 338.2874

$\mathrm{N}(\mathrm{Tc}) \sim 1$, Ea $\sim$ Light + Temperature, Eh $\sim$ Light * Temperature, Th Temperature 338.4316

$\mathrm{N}(\mathrm{Tc}) \sim$ Light + Temperature, Ea $\sim$ Light * Temperature, Eh $\sim$ Temperature, Th $\sim$ Temperature 338.5049 
$\mathrm{N}(\mathrm{Tc}) \sim 1$, Ea Temperature, Eh Light + Temperature, Th Temperature 338.7185

$\mathrm{N}(\mathrm{Tc}) \sim$ Temperature, Ea $\sim 1$, Eh Temperature, Th Light * Temperature 338.8273

$\mathrm{N}(\mathrm{Tc}) \sim$ Temperature, Ea Light, Eh Light + Temperature, Th Light 339.2201

$\mathrm{N}(\mathrm{Tc}) \sim 1$ 1, Ea Light, Eh Temperature, Th Light + Temperature 339.5326

$\mathrm{N}(\mathrm{Tc}) \sim$ Light, Ea 1, Eh Light, Th 1339.7421

$\mathrm{N}(\mathrm{Tc}) \sim 1$, Ea Light * Temperature, Eh Light + Temperature, Th $\sim 1339.7504$

$\mathrm{N}(\mathrm{Tc}) \sim$ Temperature, Ea $\sim$ Light + Temperature, Eh $\sim$ Light + Temperature, Th $\sim$ Temperature 339.8608

$\mathrm{N}(\mathrm{Tc}) \sim$ Temperature, Ea Light * Temperature, Eh 1, Th Light 340.0903

$\mathrm{N}(\mathrm{Tc}) \sim 1$, Ea Light * Temperature, Eh Light + Temperature, Th Temperature 340.1579

$\mathrm{N}(\mathrm{Tc}) \sim 1$, Ea 1, Eh 1, Th Light * Temperature 340.3486

$\mathrm{N}(\mathrm{Tc}) \sim 1$, Ea $\sim$ Light, Eh $\sim 1$, Th Light * Temperature 340.5451

$\mathrm{N}(\mathrm{Tc}) \sim$ Temperature, Ea Light + Temperature, Eh Light * Temperature, Th 1340.5582

$\mathrm{N}(\mathrm{Tc}) \sim$ Temperature, Ea 1, Eh Light, Th Light * Temperature 341.1457

$\mathrm{N}(\mathrm{Tc}) \sim$ Temperature, Ea Light + Temperature, Eh Light + Temperature, Th 1341.2757

$\mathrm{N}(\mathrm{Tc}) \sim$ Temperature, Ea $\sim 1$, Eh Light * Temperature, Th Light 341.3404

$\mathrm{N}(\mathrm{Tc}) \sim 1$, Ea $\sim$ Temperature, Eh Light * Temperature, Th $~ 1341.4933$

$\mathrm{N}(\mathrm{Tc}) \sim 1$, Ea $\sim$ Light, Eh $\sim$ Light + Temperature, Th $\sim$ Light + Temperature 341.497

$\mathrm{N}(\mathrm{Tc}) \sim 1$, Ea 1, Eh $\sim$ Temperature, Th Light + Temperature 341.5027

$\mathrm{N}(\mathrm{Tc}) \sim$ Temperature, Ea $\sim$ Light, Eh $\sim$ Light * Temperature, Th Light * Temperature 341.8181

$\mathrm{N}(\mathrm{Tc}) \sim$ Temperature, Ea $\sim$ Light, Eh $\sim$ Temperature, Th Light + Temperature 341.9956

$\mathrm{N}(\mathrm{Tc}) \sim$ Temperature, Ea Temperature, Eh Light + Temperature, Th 1343.0134

$\mathrm{N}(\mathrm{Tc}) \sim$ Temperature, Ea $\sim$ Light, Eh $\sim$ Light + Temperature, Th $\sim$ Light + Temperature 343.212

$\mathrm{N}(\mathrm{Tc}) \sim 1$, Ea $\sim$ Temperature, Eh Light * Temperature, Th Temperature 343.5551

N(Tc) Light * Temperature, Ea 1, Eh Light, Th 1343.6144

$\mathrm{N}(\mathrm{Tc}) \sim 1$, Ea Light * Temperature, Eh Light * Temperature, Th 1343.773

$\mathrm{N}(\mathrm{Tc}) \sim$ Temperature, Ea $\sim$ Temperature, Eh $\sim$ Light + Temperature, Th $\sim$ Temperature 343.8543

$\mathrm{N}(\mathrm{Tc}) \sim$ Temperature, Ea Light, Eh Light * Temperature, Th Light 343.8555

$\mathrm{N}(\mathrm{Tc}) \sim$ Light + Temperature, Ea 1, Eh $\sim$ Temperature, Th $\sim 1343.9421$

$\mathrm{N}(\mathrm{Tc}) \sim$ Light, Ea 1, Eh Light, Th Temperature 344.0668 
$\mathrm{N}(\mathrm{Tc}) \sim$ Temperature, Ea $\sim$ Light + Temperature, Eh $\sim$ Light * Temperature, Th $\sim$ Temperature 344.7419

$\mathrm{N}(\mathrm{Tc}) \sim$ Temperature, Ea $\sim 1$, Eh $\sim 1$, Th Light * Temperature 344.8638

$\mathrm{N}(\mathrm{Tc}) \sim 1$, Ea $\sim$ Light $*$ Temperature, Eh $\sim$ Light * Temperature, Th $\sim$ Temperature 344.9934 $\mathrm{N}(\mathrm{Tc}) \sim$ Temperature, Ea Light, Eh $\sim 1$, Th Light * Temperature 345.3258

$\mathrm{N}(\mathrm{Tc}) \sim 1$, Ea $\sim$ Light, Eh Light, Th Light * Temperature 345.3787 $\mathrm{N}(\mathrm{Tc}) \sim 1$, Ea $~ 1, \mathrm{Eh} \sim 1$, Th Light + Temperature 345.4259

N(Tc) 1, Ea Light, Eh 1, Th Light + Temperature 345.8094

$\mathrm{N}(\mathrm{Tc}) \sim 1$, Ea $\sim 1$, Eh $\sim$ Light, Th Light 345.9191

$\mathrm{N}(\mathrm{Tc}) \sim$ Temperature, Ea Light * Temperature, Eh Light + Temperature, Th 1345.9585

N(Tc) Light + Temperature, Ea Light, Eh Temperature, Th 1345.9769

$\mathrm{N}(\mathrm{Tc}) \sim$ Temperature, Ea $\sim$ Light * Temperature, Eh $\sim$ Light + Temperature, Th $\sim$ Temperature 346.3724

$\mathrm{N}(\mathrm{Tc}) \sim$ Light + Temperature, Ea $\sim 1$, Eh $\sim$ Temperature, Th $\sim$ Temperature 346.3963

$\mathrm{N}(\mathrm{Tc}) \sim$ Light + Temperature, Ea $\sim$ Temperature, Eh $\sim$ Temperature, Th $\sim$ Temperature 346.8634 $\mathrm{N}(\mathrm{Tc}) \sim 1$, Ea $\sim 1$, Eh 1, Th Light 346.9323

$\mathrm{N}(\mathrm{Tc}) \sim 1$, Ea $\sim$ Temperature, Eh Light, Th 1346.9574

$\mathrm{N}(\mathrm{Tc}) \sim$ Light, Ea $\sim 1$, Eh $~ 1, \mathrm{Th} \sim 1 \quad 347.0247$

$\mathrm{N}(\mathrm{Tc}) \sim 1$, Ea $\sim$ Light, Eh $\sim 1$, Th Light 347.1081

$\mathrm{N}(\mathrm{Tc}) \sim$ Temperature, Ea $\sim 1$, Eh $\sim$ Light + Temperature, Th Light + Temperature 347.1876

$\mathrm{N}(\mathrm{Tc}) \sim 1$, Ea $\sim$ Light, Eh $\sim$ Light, Th $\sim$ Light + Temperature $\quad 347.394$

$\mathrm{N}(\mathrm{Tc}) \sim$ Temperature, Ea $\sim$ Temperature, Eh Light * Temperature, Th $\sim 1347.5407$

$\mathrm{N}(\mathrm{Tc}) \sim 1$, Ea Light, Eh Light, Th Light 348.3733

$\mathrm{N}(\mathrm{Tc}) \sim$ Light, Ea $\sim$ Light, Eh $\sim 1$, Th $\sim 1348.7014$

$\mathrm{N}(\mathrm{Tc}) \sim$ Temperature, Ea $\sim$ Temperature, Eh Light * Temperature, Th $\sim$ Temperature 349.1589

$\mathrm{N}(\mathrm{Tc}) \sim 1, \mathrm{Ea} \sim 1$, Eh $\sim$ Light * Temperature, Th Light + Temperature 349.2081

$\mathrm{N}(\mathrm{Tc}) \sim$ Temperature, Ea $\sim 1$, Eh $\sim$ Light, Th Light + Temperature 349.4125

$\mathrm{N}(\mathrm{Tc}) \sim 1$, Ea Light + Temperature, Eh Light, Th 1349.538 
$\mathrm{N}(\mathrm{Tc}) \sim$ Temperature, Ea $\sim 1$, Eh $\sim 1$, Th $\sim$ Light + Temperature 349.8621 $\mathrm{N}(\mathrm{Tc}) \sim$ Temperature, Ea Light * Temperature, Eh Light * Temperature, Th 1350.3736 N(Tc) Temperature, Ea Light, Eh 1, Th Light + Temperature 350.4185 $\mathrm{N}(\mathrm{Tc}) \sim$ Temperature, Ea 1, Eh Light, Th Light 350.5185 $\mathrm{N}(\mathrm{Tc}) \sim$ Temperature, Ea $\sim 1$, Eh $\sim 1$, Th Light 351.3178

$\mathrm{N}(\mathrm{Tc}) \sim 1$, Ea $\sim$ Temperature, Eh Light, Th Temperature 351.3802 $\mathrm{N}(\mathrm{Tc}) \sim$ Light, Ea 1, Eh Temperature, Th 1351.3941

$\mathrm{N}(\mathrm{Tc}) \sim$ Temperature, Ea 1, Eh Light * Temperature, Th Light + Temperature 351.5034 $\mathrm{N}(\mathrm{Tc}) \sim 1$, Ea $\sim$ Light, Eh $\sim$ Temperature, Th Light 351.5621

$\mathrm{N}(\mathrm{Tc}) \sim$ Temperature, Ea $\sim$ Temperature, Eh Light, Th 1351.619 $\mathrm{N}(\mathrm{Tc}) \sim$ Temperature, Ea Light, Eh 1, Th Light 351.6462 $\mathrm{N}(\mathrm{Tc}) \sim 1$, Ea $\sim 1$, Eh $\sim$ Temperature, Th Light 351.7129 $\mathrm{N}(\mathrm{Tc}) \sim$ Temperature, Ea $\sim$ Light, Eh $\sim$ Light, Th Light + Temperature 352.2652 $\mathrm{N}(\mathrm{Tc}) \sim$ Light, Ea Light, Eh Temperature, Th 1353.0511 $\mathrm{N}(\mathrm{Tc}) \sim$ Temperature, Ea $\sim$ Light, Eh $\sim$ Light, Th Light 353.0951

N(Tc) Light, Ea Light, Eh 1, Th Temperature 353.4012

$\mathrm{N}(\mathrm{Tc}) \sim$ Light + Temperature, Ea $\sim$ Light * Temperature, Eh $\sim 1$, Th $\sim$ Temperature 353.5995 $\mathrm{N}(\mathrm{Tc}) \sim 1$, Ea $\sim$ Light + Temperature, Eh $\sim$ Light, Th $\sim$ Temperature 353.9968 N(Tc) Temperature, Ea Light + Temperature, Eh Light, Th 1354.3486 $\mathrm{N}(\mathrm{Tc}) \sim 1$, Ea $\sim$ Light * Temperature, Eh $\sim$ Light, Th $\sim 1354.7509$ N(Tc) Light, Ea 1, Eh Temperature, Th Temperature 354.7995 $\mathrm{N}(\mathrm{Tc}) \sim 1$, Ea $\sim$ Light, Eh $\sim$ Light + Temperature, Th 1354.8868 $\mathrm{N}(\mathrm{Tc}) \sim 1$, Ea $\sim$ Light, Eh $\sim$ Light + Temperature, Th $\sim$ Temperature 354.9669 $\mathrm{N}(\mathrm{Tc}) \sim 1$, Ea $\sim$ Light + Temperature, Eh 1, Th 355.5921

$\mathrm{N}(\mathrm{Tc}) \sim$ Temperature, Ea Light, Eh $\sim$ Temperature, Th Light 356.1079 $\mathrm{N}(\mathrm{Tc}) \sim$ Temperature, Ea $\sim 1$, Eh $\sim$ Temperature, Th Light 356.1584 $\mathrm{N}(\mathrm{Tc}) \sim$ Temperature, Ea $\sim$ Temperature, Eh $\sim$ Light, Th $\sim$ Temperature 356.325 $\mathrm{N}(\mathrm{Tc}) \sim$ Light, Ea $\sim$ Light, Eh $\sim$ Temperature, Th $\sim$ Temperature 356.9616 $\mathrm{N}(\mathrm{Tc}) \sim$ Light + Temperature, Ea Light, Eh 1, Th $\sim$ Temperature 357.0855 
$\mathrm{N}(\mathrm{Tc}) \sim$ Temperature, Ea Light + Temperature, Eh 1, Th 1360.1858

$\mathrm{N}(\mathrm{Tc}) \sim$ Temperature, Ea Light, Eh Light + Temperature, Th $\sim$ Temperature 360.3689 $\mathrm{N}(\mathrm{Tc}) \sim 1$, Ea $\sim$ Temperature, Eh Temperature, Th 1360.397

$\mathrm{N}(\mathrm{Tc}) \sim 1$, Ea $\sim$ Light + Temperature, Eh $\sim 1$, Th $\sim$ Temperature 360.5872 $\mathrm{N}(\mathrm{Tc}) \sim$ Temperature, Ea $\sim$ Temperature, Eh $\sim 1$, Th $\sim 1361.5556$

$\mathrm{N}(\mathrm{Tc}) \sim$ Light, Ea $\sim$ Light + Temperature, Eh $\sim 1$, Th $\sim$ Temperature 361.7121 $\mathrm{N}(\mathrm{Tc}) \sim 1$, Ea $\sim$ Temperature, Eh 1, Th Temperature 361.9405

$\mathrm{N}(\mathrm{Tc}) \sim$ Temperature, Ea Light, Eh Light * Temperature, Th Temperature 363.0164

$\mathrm{N}(\mathrm{Tc}) \sim 1$, Ea $\sim$ Light * Temperature, Eh $\sim$ Temperature, Th $\sim 1363.6273$

$\mathrm{N}(\mathrm{Tc}) \sim 1$, Ea $\sim$ Light + Temperature, Eh $\sim$ Temperature, Th $\sim$ Temperature 364.1207

$\mathrm{N}(\mathrm{Tc}) \sim$ Temperature, Ea Light + Temperature, Eh Temperature, Th 1364.1488

$\mathrm{N}(\mathrm{Tc}) \sim$ Temperature, Ea Light * Temperature, Eh 1, Th 1365.1245

$\mathrm{N}(\mathrm{Tc}) \sim$ Temperature, Ea $\sim$ Light * Temperature, Eh $\sim$ Light, Th $\sim$ Temperature 365.1543

$\mathrm{N}(\mathrm{Tc}) \sim$ Temperature, Ea Temperature, Eh Temperature, Th 1365.2319

$\mathrm{N}(\mathrm{Tc}) \sim$ Temperature, Ea $\sim$ Light + Temperature, Eh 1, Th Temperature 365.4657

$\mathrm{N}(\mathrm{Tc}) \sim 1$, Ea Light * Temperature, Eh 1, Th Temperature 365.5549

$\mathrm{N}(\mathrm{Tc}) \sim$ Temperature, Ea $\sim$ Temperature, Eh $\sim 1$, Th $\sim$ Temperature 366.6657

$\mathrm{N}(\mathrm{Tc}) \sim$ Light, Ea $\sim$ Light * Temperature, Eh $\sim 1$, Th Temperature 366.6926

$\mathrm{N}(\mathrm{Tc}) \sim 1, \mathrm{Ea} \sim 1, \mathrm{Eh} \sim$ Light + Temperature, Th 1368.7992

$\mathrm{N}(\mathrm{Tc}) \sim$ Temperature, Ea $\sim$ Light * Temperature, Eh $\sim$ Temperature, Th $\sim 1369.1651$

$\mathrm{N}(\mathrm{Tc}) \sim 1, \mathrm{Ea} \sim$ Light * Temperature, Eh $\sim$ Temperature, Th $\sim$ Temperature 369.1664 $\mathrm{N}(\mathrm{Tc}) \sim 1$, Ea $\sim$ Temperature, Eh $\sim$ Temperature, Th $\sim$ Temperature 369.1856 
$\mathrm{N}(\mathrm{Tc}) \sim$ Temperature, Ea $\sim$ Light + Temperature, Eh $\sim$ Temperature, Th $\sim$ Temperature 369.5038 $\mathrm{N}(\mathrm{Tc}) \sim 1$, Ea $\sim$ Light, Eh Light * Temperature, Th 1370.6122 $\mathrm{N}(\mathrm{Tc}) \sim$ Temperature, Ea Light * Temperature, Eh $\sim 1$, Th $\sim$ Temperature 370.7988 $\mathrm{N}(\mathrm{Tc}) \sim$ Light + Temperature, Ea Light, Eh 1, Th 1372.2907 $\mathrm{N}(\mathrm{Tc}) \sim 1$, Ea $\sim 1$, Eh Light, Th 1372.705

$\mathrm{N}(\mathrm{Tc}) \sim$ Temperature, Ea $\sim$ Light * Temperature, Eh $\sim$ Light * Temperature, Th $\sim$ Temperature 373.8418 $\mathrm{N}(\mathrm{Tc}) \sim$ Temperature, Ea $\sim$ Temperature, Eh $\sim$ Temperature, Th $\sim$ Temperature 374.1744

$\mathrm{N}(\mathrm{Tc}) \sim 1$, Ea $\sim 1$, Eh $\sim$ Light + Temperature, Th $\sim$ Temperature 374.606 $\mathrm{N}(\mathrm{Tc}) \sim 1$, Ea Light, Eh Light, Th 1374.9695

$\mathrm{N}(\mathrm{Tc}) \sim$ Temperature, Ea $\sim$ Light * Temperature, Eh $\sim$ Temperature, Th $\sim$ Temperature 375.0474

$\mathrm{N}(\mathrm{Tc}) \sim$ Temperature, Ea $\sim 1$, Eh $\sim$ Light + Temperature, Th 1376.3268 $\mathrm{N}(\mathrm{Tc}) \sim 1$, Ea $\sim 1$, Eh $\sim$ Light, Th $\sim$ Temperature 376.8054 N(Tc) Temperature, Ea Light, Eh Light + Temperature, Th 1377.0801 $\mathrm{N}(\mathrm{Tc}) \sim$ Temperature, Ea $\sim 1$, Eh $\sim$ Light, Th 1377.0968 $\mathrm{N}(\mathrm{Tc}) \sim 1$, Ea $\sim 1$, Eh Light * Temperature, Th 1377.3256

$\mathrm{N}(\mathrm{Tc}) \sim$ Temperature, Ea $~ 1$, Eh $\sim$ Light + Temperature, Th $\sim$ Temperature 377.5984 $\mathrm{N}(\mathrm{Tc}) \sim 1$, Ea $\sim 1$, Eh 1, Th 1378.1854

$\mathrm{N}(\mathrm{Tc}) \sim 1$, Ea $\sim$ Light, Eh $\sim$ Light, Th $\sim$ Temperature 379.0559 $\mathrm{N}(\mathrm{Tc}) \sim 1$, Ea $\sim 1$, Eh $\sim$ Temperature, Th $\sim 1379.2271$ $\mathrm{N}(\mathrm{Tc}) \sim 1$, Ea $\sim$ Light, Eh 1, Th 1379.445

N(Tc) $\sim$ Temperature, Ea Light, Eh Light, Th 1379.4984

$\mathrm{N}(\mathrm{Tc}) \sim 1$, Ea $\sim 1$, Eh $\sim$ Light * Temperature, Th $\sim$ Temperature 379.5335

$\mathrm{N}(\mathrm{Tc}) \sim 1$, Ea $\sim$ Light, Eh $\sim$ Temperature, Th 1380.0629

$\mathrm{N}(\mathrm{Tc}) \sim$ Light + Temperature, Ea $\sim 1$, Eh $\sim 1$, Th $\sim$ Temperature 381.0925 $\mathrm{N}(\mathrm{Tc}) \sim$ Temperature, Ea $~ 1$, Eh Light, Th Temperature 381.4489

$\mathrm{N}(\mathrm{Tc}) \sim$ Temperature, Ea $\sim 1$, Eh $\sim$ Light * Temperature, Th 1381.5215

$\mathrm{N}(\mathrm{Tc}) \sim 1$, Ea $\sim 1$, Eh $\sim$ Temperature, Th $\sim$ Temperature 381.5992

$\mathrm{N}(\mathrm{Tc}) \sim$ Temperature, Ea $\sim 1$, Eh $\sim 1$, Th $~ 1382.3585$

$\mathrm{N}(\mathrm{Tc}) \sim$ Temperature, Ea $\sim$ Light, Eh $\sim$ Light * Temperature, Th 1382.4813 
$\mathrm{N}(\mathrm{Tc}) \sim 1$, Ea $\sim 1$, Eh 1, Th Temperature 382.4827 $\mathrm{N}(\mathrm{Tc}) \sim$ Temperature, Ea $\sim 1$, Eh $\sim$ Temperature, Th 1382.5039 $\mathrm{N}(\mathrm{Tc}) \sim 1$, Ea Light, Eh $\sim$ Temperature, Th Temperature 382.7764 $\mathrm{N}(\mathrm{Tc}) \sim$ Temperature, Ea Light, Eh $\sim$ Temperature, Th $\sim 1383.3059$ $\mathrm{N}(\mathrm{Tc}) \sim$ Temperature, Ea Light, Eh $\sim 1$, Th $~ 1383.7582$

$\mathrm{N}(\mathrm{Tc}) \sim$ Temperature, Ea $\sim 1$, Eh $\sim$ Light * Temperature, Th $\sim$ Temperature 383.808 $\mathrm{N}(\mathrm{Tc}) \sim$ Temperature, Ea $\sim$ Light, Eh $\sim$ Light, Th $\sim$ Temperature 383.8509 $\mathrm{N}(\mathrm{Tc}) \sim 1$, Ea $\sim$ Light, Eh $\sim 1$, Th Temperature 383.9215

$\mathrm{N}(\mathrm{Tc}) \sim$ Temperature, Ea $\sim 1$, Eh $\sim$ Temperature, Th $\sim$ Temperature 384.9088 $\mathrm{N}(\mathrm{Tc}) \sim$ Temperature, Ea $\sim$ Light, Eh $\sim$ Temperature, Th $\sim$ Temperature 386.0658 $\mathrm{N}(\mathrm{Tc}) \sim$ Temperature, Ea $\sim 1$, Eh $\sim 1$, Th $\sim$ Temperature 386.8926 $\mathrm{N}(\mathrm{Tc}) \sim$ Temperature, Ea Light, Eh 1, Th Temperature 388.4849 $\mathrm{N}(\mathrm{Tc}) \sim$ Light, Ea $\sim 1$ Eh $\sim 1$, Th $\sim$ Temperature 390.896 $\mathrm{N}(\mathrm{Tc}) \sim$ Light + Temperature, Ea $\sim$ Light * Temperature, Eh $\sim$ Temperature, Th $\sim$ Light * Temperature $\quad$ NA $\mathrm{N}(\mathrm{Tc}) \sim$ Light * Temperature, Ea $\sim$ Light + Temperature, Eh $\sim$ Temperature, Th $\sim$ Light * Temperature NA $\mathrm{N}(\mathrm{Tc}) \sim$ Light $*$ Temperature, Ea $\sim$ Light + Temperature, Eh $\sim$ Light + Temperature, Th $\sim$ Light * Temperature $\quad$ NA $\mathrm{N}(\mathrm{Tc}) \sim$ Light * Temperature, Ea $\sim$ Light + Temperature, Eh $\sim$ Light * Temperature, Th $\sim$ Light * Temperature $\quad$ NA $\mathrm{N}(\mathrm{Tc}) \sim$ Light $*$ Temperature, Ea Light * Temperature, Eh $\sim$ Temperature, Th $\sim$ Light * Temperature $\quad$ NA $\mathrm{N}(\mathrm{Tc}) \sim$ Light * Temperature, Ea $\sim$ Light * Temperature, Eh $\sim$ Light + Temperature, Th $\sim$ Light * Temperature $\quad$ NA $\mathrm{N}(\mathrm{Tc}) \sim$ Light * Temperature, Ea $\sim$ Light * Temperature, Eh Light * Temperature, Th $\sim$ Light 


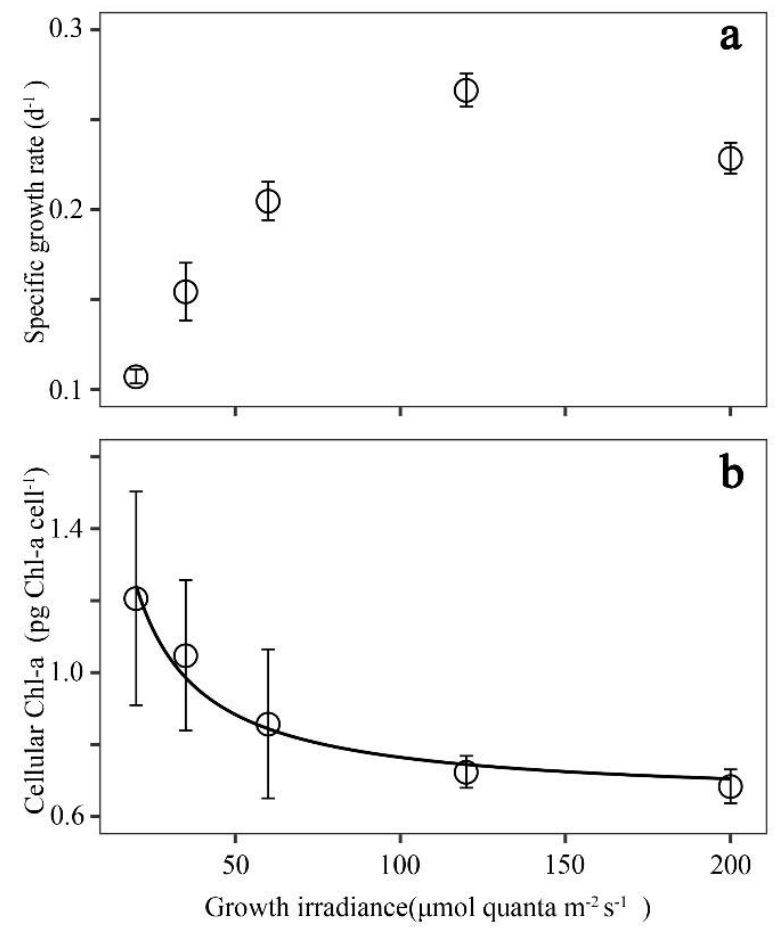

Figure S1. (a) Specific growth rate and (b) cellular Chl-a content of Trichodesmium grown across a range of light intensity. Values represent the means \pm standard deviations of biological replicates $(n=3)$. Cultures were grown at $25^{\circ} \mathrm{C}$ without aeration. In panel (b), the curve gives the best fit using inverse proportional function: $\mathrm{y}=11.9233 \mathrm{x}+$ 0.6451 . 


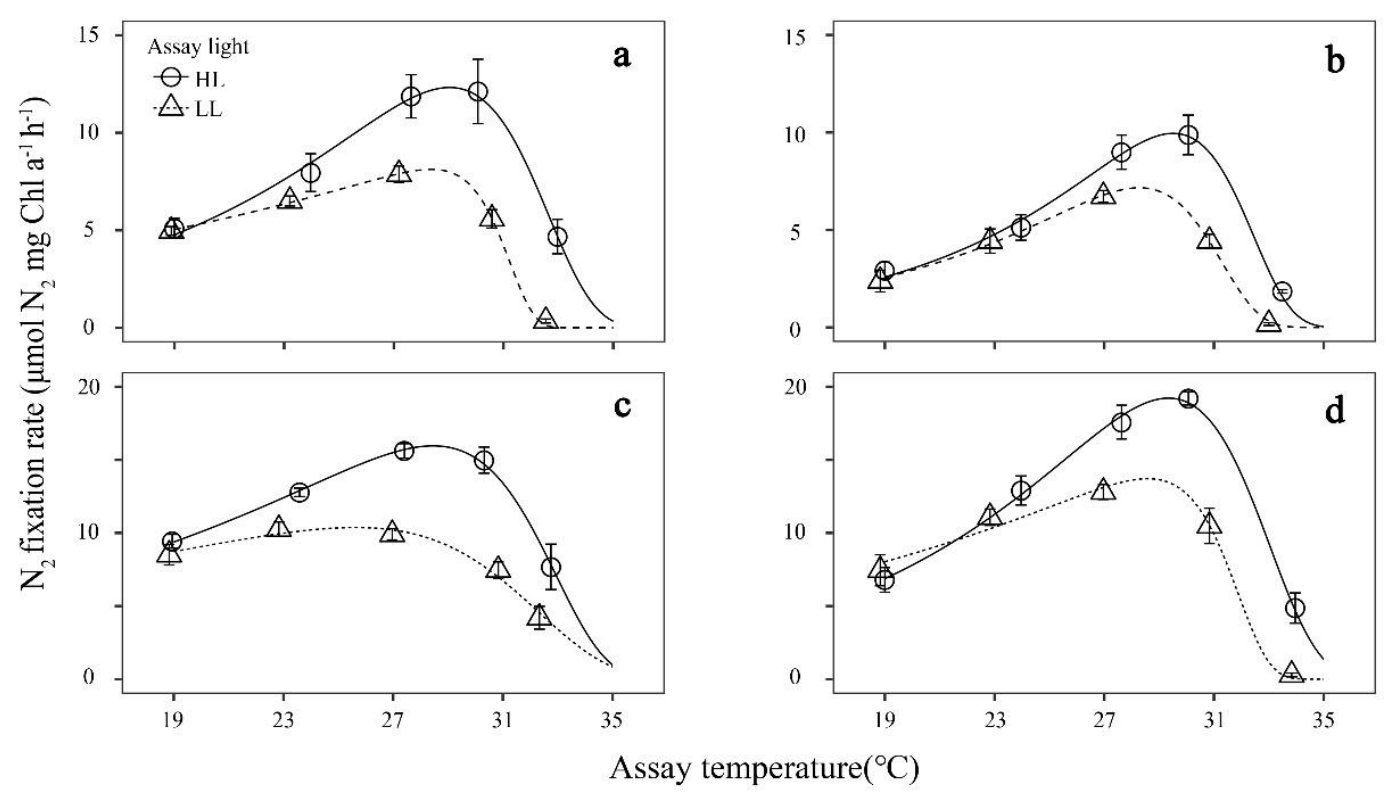

Figure S2. Short-term temperature norms of $\mathrm{N}_{2}$ fixation measured at two light intensity (HL: $160 \mu \mathrm{mol}$ quanta $\mathrm{m}^{-2} \mathrm{~s}^{-1}$, LL: $50 \mu \mathrm{mol}$ quanta $\mathrm{m}^{-2} \mathrm{~s}^{-1}$ ) in Trichodesmium acclimated to: (a) $23{ }^{\circ} \mathrm{C}$ and $\mathrm{LL}$, (b) $27^{\circ} \mathrm{C}$ and $\mathrm{LL}$, (c) $23^{\circ} \mathrm{C}$ and $\mathrm{HL}$, (d) $27^{\circ} \mathrm{C}$ and HL. Values represent the means \pm standard deviations of biological replicates $(n=3)$. Fitted lines are based on mean parameters at each treatment across replicates $(n=3)$ derived from nonlinear least squares regression using the modified Sharpe-Schoolfield model (function 'nlm' in package 'stats' in R). 